\title{
Multiscale Image Representation and Texture Extraction Using Hierarchical Variational Decomposition
}

\author{
Liming Tang ${ }^{1,2}$ and Chuanjiang $\mathrm{He}^{1}$ \\ ${ }^{1}$ College of Mathematics and Statistics, Chongqing University, Chongqing 401331, China \\ ${ }^{2}$ College of Mathematics and Physics, Chongqing University of Science and Technology, Chongqing 401331, China \\ Correspondence should be addressed to Liming Tang; tlmcs78@foxmail.com
}

Received 19 April 2013; Revised 1 July 2013; Accepted 30 July 2013

Academic Editor: Ke Chen

Copyright (c) 2013 L. Tang and C. He. This is an open access article distributed under the Creative Commons Attribution License, which permits unrestricted use, distribution, and reproduction in any medium, provided the original work is properly cited.

\begin{abstract}
In order to achieve a mutiscale representation and texture extraction for textured image, a hierarchical $\left(B V, G_{p}, L^{2}\right)$ decomposition model is proposed in this paper. We firstly introduce the proposed model which is obtained by replacing the fixed scale parameter of the original $\left(B V, G_{p}, L^{2}\right)$ decomposition with a varying sequence. And then, the existence and convergence of the hierarchical decomposition are proved. Furthermore, we show the nontrivial property of this hierarchical decomposition. Finally, we introduce a simple numerical method for the hierarchical decomposition, which utilizes gradient decent for energy minimization and finite difference for the associated gradient flow equations. Numerical results show that the proposed hierarchical $\left(B V, G_{p}, L^{2}\right)$ decomposition is very appropriate for multiscale representation and texture extraction of textured image.
\end{abstract}

\section{Introduction}

A grayscale image can be represented by a function $f:(x, y) \in$ $\Omega \rightarrow \mathbb{R}$ with $f \in L^{2}(\Omega)$, where $\Omega$ is an open, bounded, and connected subset of $\mathbb{R}^{2}$, typically a rectangle or a square $[1,2]$. We are interested in the decomposition of $f$ into two components, $f=u+v$ [3-5], or three components, $f=u+$ $v+r$ [6-9], where $u$ represents piecewise-smooth (cartoon or structure) component of $f$ and $v$ represents the oscillatory component of $f$, that is, texture, and $r$ represents the residual (noise). Image decomposition is an important image processing task, which is widely used in image denoising [4, 10, $11]$, deblurring $[12,13]$, image representation $[5,13]$, texture extraction or discrimination $[6,14]$, and so on. It has seen much recent progress, much of which has particularly been made through the use of variational framework to model oscillatory component that represents texture; see, for example, $[2-6,8-14]$. We give here some classical examples of image decomposition models by variational approaches that are most related to our present work.

A celebrated decomposition easier to implement is the total variation (TV) minimization model by Rudin, Osher, and Fatemi (ROF) [3] for image denoising, in which an image $f \in L^{2}(\Omega)$ is split into $u \in B V(\Omega)$ and $v \in L^{2}(\Omega)$ :

$$
\begin{aligned}
& (u, v) \\
& \quad=\arg \inf \left\{J(f ; u, v)=|u|_{B V(\Omega)}+\mu\|v\|_{L^{2}(\Omega)}, f=u+v\right\},
\end{aligned}
$$

which yields so-called $\left(B V, L^{2}\right)$ decomposition. This model is convex and easy to solve in practice. The function $u \in B V(\Omega)$ allows for discontinuities along curves; therefore, edges and contours are preserved in the restored image $u$.

However, as Meyer pointed out in [15], the function space $L^{2}(\Omega)$ is not the most suitable one to model oscillatory components, since the oscillatory functions do not have small $L^{2}$-norms. He suggested using $(B V(\Omega))^{\prime}$, the dual space of $B V(\Omega)$, instead of $L^{2}(\Omega)$ for the oscillatory components. However, there is no known integral representation of continuous linear functional on $B V(\Omega)$. To address this problem, Meyer used another slightly larger space to approximate $(B V(\Omega))^{\prime}$. Using $G(\Omega)$ to characterize oscillatory components 
yields the $(B V, G)$ decomposition by solving the following variational problem:

$$
\inf _{u \in B V(\Omega), v \in G(\Omega)}\left\{|u|_{B V(\Omega)}+\mu\|v\|_{G(\Omega)}, f=u+v\right\} .
$$

The $(B V, G)$ decomposition model can better extract texture; however, it cannot be directly solved in practice due to the nature of the $G$-norm $[4,6,14]$, for which there is no standard calculation of the associated Euler-Lagrange equation. Vese and Osher $[6,14]$ first overcame this difficulty by replacing the space $G(\Omega)$ with $G_{p}(\Omega)(p \geq 1)$. Then, the $(B V, G)$ decomposition model (2) is approximated by the following minimization problem:

$$
\inf _{u \in B V(\Omega), v \in G_{p}(\Omega)}\left\{|u|_{B V(\Omega)}+\mu\|v\|_{G_{p}(\Omega)}, f=u+v\right\} .
$$

In [6], Vese and Osher did not solve (3) directly but adapted the model by adding a fidelity term into the energy functional to guarantee $f \approx u+v$. In detail, their variational formulation is defined as

$$
\inf _{u \in B V(\Omega), v \in G_{p}(\Omega)}\left\{|u|_{B V(\Omega)}+\lambda\|f-u-v\|_{L^{2}(\Omega)}^{2}+\mu\|v\|_{G_{p}(\Omega)}\right\} .
$$

In this $\left(B V, G_{p}, L^{2}\right)$ decomposition, the image $f$ is discomposed into three components, $f=u+v+r$ with $u \in B V(\Omega)$, $v \in G_{p}(\Omega)$, and $r \in L^{2}(\Omega)$.

The previous models are examples of a larger class of the fixed scale decompositions (the scale parameters in these models are fixed). It has been argued that a human visualizes a scene in multiple scales $[16,17]$. Then, multiscale approaches are appropriate for image representation because a single scale may not be a perfect simulation of the human visual perception. In order to achieve reliable image information in different scales, both the large-scale and small-scale behaviors should be investigated and incorporated appropriately. Thus, a natural way to address this problem is the multiscale analysis.

Tadmor et al. $[5,13]$ presented a hierarchical decomposition based on the ROF model (1) to achieve multiscale image representation, in which the scale parameter is not fixed, but a varying sequence: starting with an initial scale $\mu_{0}$,

$$
\begin{gathered}
f=u_{0}+v_{0}, \\
\left(u_{0}, v_{0}\right)=\arg \inf \left\{|u|_{B V(\Omega)}+\mu_{0}\|v\|_{L^{2}(\Omega)}^{2}, f=u+v\right\},
\end{gathered}
$$

and then, successive application of the following dyadic refinement step

$$
\begin{gathered}
v_{i}=u_{i+1}+v_{i+1}, \\
\left(u_{i+1}, v_{i+1}\right)=\arg \inf \left\{|u|_{B V(\Omega)}\right. \\
\left.\quad+\mu_{0} 2^{i+1}\|v\|_{L^{2}(\Omega)}^{2}, v_{i}=u+v\right\}
\end{gathered}
$$

produces, after $k$ such steps, the hierarchical $\left(B V, L^{2}\right)$ decomposition of $f$ :

$$
f=\sum_{i=0}^{k} u_{i}+v_{k}, \quad(k=0,1, \ldots) .
$$

In this study, we focus on multiscale representation and texture extraction for textured image. As discussed previously, the $\left(B V, L^{2}\right)$ decomposition is not the best one for textured image, so using hierarchical $\left(B V, L^{2}\right)$ decomposition (7) introduced by Tadmor et al. to implement multiscale representation and texture extraction for textured image is obviously not the best choice. We thus in this paper propose the hierarchical decomposition using the $\left(B V, G_{p}, L^{2}\right)$ model (4), which enables us to capture an intermediate regularity between $L^{2}(\Omega)$ and $B V(\Omega)$ and oscillation between $L^{2}(\Omega)$ and $G_{p}(\Omega)$. We here adopt $\left(B V, G_{p}, L^{2}\right)$ decomposition because $G_{p}(\Omega)$ is a very suitable function space to model oscillatory patterns $[6,14]$; in addition, the $G_{p}$-norm is easier to solve in practice. In the proposed hierarchical $\left(B V, G_{p}, L^{2}\right)$ decomposition, the scale parameter is not fixed but varies over a sequence of dyadic scales. Consequently, the decomposition of a textured image is not predetermined but is resolved in terms of layers of intermediate scales. So, we can achieve multiscale image representation. Compared to Tadmor et al's 2-tuple hierarchical decomposition, the proposed 3-tuple hierarchical decomposition can precisely extract texture in different scales.

\section{Preliminaries}

So far, there have been a lot of efficient variational decomposition models for textured image, much of which follows Meyer's work. The $\left(B V, G_{p}, L^{2}\right)$ decomposition introduced by Vese and Osher is the first one to practically solve the Meyer's $(B V, G)$ model presented in (2), in which cartoon component is measured in $B V(\Omega)$ and texture component in $G_{p}(\Omega)$, instead of $G(\Omega)$. We here recall the definition and some known results of $B V(\Omega), G(\Omega)$ and $G_{p}(\Omega)$, which are much related to our present study.

Definition 1. Let $\Omega \subset \mathbb{R}^{2}$ be an open subset with Lipschitz boundary. Then, $B V(\Omega)$ is the subspace of $L^{1}(\Omega)$ such that the following quantity

$$
\begin{aligned}
&|u|_{B V(\Omega)}= \int_{\Omega}|D u| \\
&= \sup \left\{\int_{\Omega} u \operatorname{div}(\varphi) d \mathbf{x} \mid \varphi \in C_{c}^{1}\left(\Omega, R^{2}\right),\right. \\
&\left.\|\varphi\|_{L^{\infty}} \leq 1\right\}
\end{aligned}
$$

is finite. Further, $\|u\|_{B V(\Omega)}=\|u\|_{L^{1}(\Omega)}+|u|_{B V(\Omega)}$ is called the $B V$-norm.

Remark 2. $B V(\Omega)$ with the norm of $\|u\|_{B V(\Omega)}$ is a Banach space, but one does not use this norm since it possesses no good compactness property. Classically, in $B V(\Omega)$ one works with the $B V$-weak ${ }^{*}$ topology, which is defined as $u_{n}$ convergence to $u$ in $B V$-weak ${ }^{*}$ topology if and only if $u_{n}$ converges to $u$ strongly in $L^{1}(\Omega)$ and $\int_{\Omega} \varphi D u_{n}$ converge to $\int_{\Omega} \varphi D u$ for all $\varphi$ in $C_{c}\left(\Omega, \mathbb{R}^{2}\right)$. 
Theorems 3 and 4 show the compactness and lower semicontinuity of $B V(\Omega)$.

Theorem 3 (see [18]). If $u_{n}$ is a uniformly bounded sequence in $B V(\Omega)$, then there exist a subsequence $u_{n_{k}}$ and $u$ in $B V(\Omega)$ such that $u_{n_{k}}$ converge to $u$ in the $B V$-weak ${ }^{*}$ topology.

Theorem 4 (see $[19,20]$ ). For $u_{n} \in B V(\Omega)$, if there exists $u \in$ $B V(\Omega)$ such that $u_{n}$ converge to $u$ in the $B V$-weak ${ }^{*}$ topology, then $|u|_{B V} \leq \underline{\lim }_{n \rightarrow+\infty}\left|u_{n}\right|_{B V}$.

Definition 5. $G(\Omega)$ consists of distributions $v$ which can be written as

$$
\begin{gathered}
v=\partial_{1} g_{1}+\partial_{2} g_{2}=\operatorname{div}(\mathbf{g}), \quad \mathbf{g} \in L^{\infty}\left(\Omega ; R^{2}\right), \\
\mathbf{g} \cdot \mathbf{n}=0 \quad \text { on } \partial \Omega,
\end{gathered}
$$

endowed with the norm

$$
\|v\|_{G(\Omega)}=\inf \left\{\|\mathbf{g}\|_{L^{\infty}(\Omega)} \mid v=\operatorname{div}(\mathbf{g}), \mathbf{g} \cdot \mathbf{n}=0 \text { on } \partial \Omega\right\} .
$$

Definition 6. $G_{p}(\Omega)$ consists of distributions $v$ which can be written as

$$
\begin{gathered}
v=\partial_{1} g_{1}+\partial_{2} g_{2}=\operatorname{div}(\mathbf{g}), \quad \mathbf{g} \in L^{p}\left(\Omega ; R^{2}\right), \\
\mathbf{g} \cdot \mathbf{n}=0 \quad \text { on } \partial \Omega,
\end{gathered}
$$

endowed with the norm

$$
\|v\|_{G_{p}(\Omega)}=\inf \left\{\|\mathbf{g}\|_{L^{p}(\Omega)} \mid v=\operatorname{div}(\mathbf{g}), \mathbf{g} \cdot \mathbf{n}=0 \text { on } \partial \Omega\right\} .
$$

For every $1 \leq p<\infty$, the space $G_{p}(\Omega)$ above can be identified with the space $W^{-1, p}(\Omega)$, the dual space to the Sobolev space $W_{0}^{1, q}(\Omega):=\left\{u: \nabla u \in L^{q}(\Omega)^{2}, u \equiv 0\right.$ on $\left.\partial \Omega\right\}$, where $1 / p+1 / q=1$. In fact, the norm $\|v\|_{G_{p}(\Omega)}$ is a dual norm to the Sobolev norm $\|\nabla u\|_{q}$. And the space $G(\Omega)=W^{-1, \infty}(\Omega)$ which is the dual to the space $W_{0}^{1,1}(\Omega)$. Moreover, if $p \rightarrow \infty$, the spaces $G_{p}(\Omega)$ approximate the space $G(\Omega)$. By the Sobolev imbedding theorems, we obtain that $\|v\|_{G_{p}(\Omega)} \leq C_{\Omega}\|v\|_{G(\Omega)}$, where $C_{\Omega}$ is a constant which is independent of $v$ but $\Omega$. So, for any $1 \leq p<\infty$, these are larger spaces than $G(\Omega)$ and allow for different choices of weaker norms for the oscillatory component $v$.

For instance, consider the sequence of one-dimensional functions $v_{n}(x)=\cos (n x)$ defined on $\Omega=[0, \pi / 2]$. Then, $v_{n}(x)=g_{n}^{\prime}(x)$, where $g_{n}(x)=(1 / n) \sin (n x)+c$. It is easy to check that

(1) $\left\|v_{n}\right\|_{L^{2}(\Omega)}=\left(\int_{0}^{\pi / 2} \cos ^{2}(n x) d x\right)^{1 / 2}=(\sqrt{\pi} / 2)>0$;

(2) $\left\|v_{n}\right\|_{G(\Omega)}=(1 / n) \rightarrow 0$ as $n \rightarrow \infty$;

(3) $\left\|v_{n}\right\|_{G_{p}(\Omega)} \quad=\quad\left(\int_{0}^{\pi / 2}\left|g_{n}(x)\right|^{p} d x\right)^{1 / p}=$ $\left(\left(1 / n^{p}\right) \int_{0}^{\pi / 2}|\sin (n x)|^{p} d x\right)^{1 / p} \leq(\pi / 2)^{1 / p}(1 / n) \rightarrow 0$ as $n \rightarrow \infty$.
This simple example demonstrates that an oscillatory function has a small $G$-norm as well as $G_{p}$-norm which both approach to zero as the frequency of oscillations increases, but importantly, not with a so small $L^{2}$-norm. So, $G$-norm and $G_{p}$-norm are more suitable than $L^{2}$-norm to measure textures in image decomposition. In addition, $G_{p}$-norm is weaker than $G$-norm. So using $G_{p}$-norm to measure oscillatory functions, we also can exactly capture the texture in the energy minimization process.

For the space $G_{p}(\Omega)$, we have the following results which will be used in what follows.

Proposition 7 (see [6]). If $v \in G_{p}(\Omega)$, then there exists $\mathbf{g} \in$ $L^{p}\left(\Omega ; \mathbb{R}^{2}\right)$ with $v=\operatorname{div}(\mathbf{g})$ and $\mathbf{g} \cdot \mathbf{n}=0$ on $\partial \Omega$, such that $\|v\|_{G_{p}(\Omega)}=\|\mathbf{g}\|_{L^{p}(\Omega)}$.

Proposition 8. If $v \in G_{p}(\Omega)$, then $\int_{\Omega} v d \mathbf{x}=0$. Indeed, $\int_{\Omega} v d \mathbf{x}=\int_{\Omega} \operatorname{div}(\mathbf{g}) d \mathbf{x}=\int_{\partial \Omega} \mathbf{g} \cdot \mathbf{n} d S=0$.

Replacing $G(\Omega)$ with $G_{p}(\Omega)(p \geq 1)$, Vese and Osher introduce the following convex minimization problem; that is, $\left(B V, G_{p}, L^{2}\right)$ decomposition:

$$
\inf _{u \in B V(\Omega), v \in G_{p}(\Omega)}\left\{|u|_{B V(\Omega)}+\lambda\|f-u-v\|_{L^{2}(\Omega)}^{2}+\mu\|v\|_{G_{p}(\Omega)}\right\},
$$

where $\lambda, \mu>0$ are tuning parameters. The first term insures that $u \in B V(\Omega)$, the second gives us $f \approx u+v$, while the third term is a penalty on the norm in $G_{p}(\Omega)$ of $v$. Clearly, if $\lambda \rightarrow \infty$ and $p \rightarrow \infty$, this model is formally an approximation of the $(B V, G)$ model (2) originally proposed by Meyer in [15].

In what follows, to simplify the notations, we always write $B V, G_{p}$, and $L^{2}$ instead of $B V(\Omega), G_{p}(\Omega)$, and $L^{2}(\Omega)$, respectively.

\section{The Proposed Hierarchical Decomposition}

3.1. Description of Hierarchical Decomposition. We firstly modify the original $\left(B V, G_{p}, L^{2}\right)$ decomposition presented in (4) to a single parameter pattern with a constraint condition $\int_{\Omega} u=\int_{\Omega} f$. The new decomposition is defined as

$$
\begin{aligned}
& \left(u_{\lambda}, v_{\lambda}\right) \\
& =\operatorname{arginf}\left\{E_{\lambda}(f, \lambda ; u, v)=|u|_{B V}+\lambda\|f-u-v\|_{L^{2}}^{2}\right. \\
& \left.+\|v\|_{G_{p}}, \int_{\Omega} u=\int_{\Omega} f\right\} .
\end{aligned}
$$

Here, the constraint condition ensures that the sum of texture $v$ and residual (noise) $r=f-u-v$ has zero mean. In this study, the parameter $\lambda$ in (14) is viewed as a scale factor which can be used to measure the scale of the extracted cartoon, especially texture. If the $\lambda$ value is too small, then only the small scale feature (coarser texture) is allocated in $v_{\lambda}$, while most of the large scale feature (smoother texture) is swept 
into the residual component $r_{\lambda}=f-\left(u_{\lambda}+v_{\lambda}\right)$. If $\lambda$ is too large, however, all the textures are extracted indiscriminately, regardless of their distinct scales.

To achieve multiscale description of a textured image, we here propose a hierarchical decomposition based on (14), which enables us to effectively extract textures in different scales.

For a given scale $\lambda$, the minimizer of $E_{\lambda}(f, \lambda ; u, v)$ is interpreted as a decomposition, $f=u_{\lambda}+v_{\lambda}+r_{\lambda}$, such that $v_{\lambda}$ captures textures in the scale $\lambda$, while the textures above $\lambda$ remain unresolved in $r_{\lambda}$. The residual $r_{\lambda}$ still consists of significant textures when viewed under a larger scale than $\lambda$, say $2 \lambda$ :

$$
r_{\lambda}=u_{2 \lambda}+v_{2 \lambda}+r_{2 \lambda}
$$

with

$$
\begin{aligned}
\left(u_{2 \lambda}, v_{2 \lambda}\right)=\operatorname{arginf}\left\{E_{\lambda}\left(r_{\lambda}, 2 \lambda ; u, v\right)=|u|_{B V}\right. \\
+2 \lambda\left\|r_{\lambda}-u-v\right\|_{L^{2}}^{2} \\
\left.+\|v\|_{G_{p}}, \int_{\Omega} u=\int_{\Omega} r_{\lambda}\right\},
\end{aligned}
$$

where $v_{2 \lambda}$ captures textures in the scale $2 \lambda$, while the textures above $2 \lambda$ remain unresolved in $r_{2 \lambda}$. The process of (15) can be continued to capture the missing large scale textures.

The proposed hierarchical decomposition can be stated as follows:

starting with an initial scale $\lambda=\lambda_{0}$,

$$
f=u_{0}+v_{0}+r_{0}
$$

where

$$
\begin{gathered}
\left(u_{0}, v_{0}\right)=\operatorname{arginf}\left\{E_{\lambda}\left(f, \lambda_{0} ; u, v\right)\right. \\
=|u|_{B V}+\lambda_{0}\|f-u-v\|_{L^{2}}^{2}+\|v\|_{G_{p}}, \\
\left.\quad \int_{\Omega} u=\int_{\Omega} f\right\} .
\end{gathered}
$$

Proceeding with successive applications of the dyadic refinement step (15), we have

$$
r_{i}=u_{i+1}+v_{i+1}+r_{i+1}, \quad i=0,1, \ldots,
$$

where

$$
\begin{gathered}
\left(u_{i+1}, v_{i+1}\right)=\arg \inf \left\{E_{\lambda}\left(r_{i}, \lambda_{0} 2^{i+1} ; u, v\right)=|u|_{B V}\right. \\
+\lambda_{0} 2^{i+1}\left\|r_{i}-u-v\right\|_{L^{2}}^{2}+\|v\|_{G_{p}}, \\
\left.\int_{\Omega} u=\int_{\Omega} r_{i}\right\} .
\end{gathered}
$$

From (19), we obtain, after $k$ such steps, the hierarchical decomposition of $f$ as follows:

$$
\begin{aligned}
f & =u_{0}+v_{0}+r_{0} \\
& =u_{0}+u_{1}+v_{0}+v_{1}+r_{1}=\cdots \\
& =u_{0}+u_{1}+\cdots+u_{k}+v_{0}+v_{1}+\cdots+v_{k}+r_{k} .
\end{aligned}
$$

The partial sum, $\sum_{i=0}^{k}\left(u_{i}+v_{i}\right)$, provides a multiscale representation of $f$, in which $\sum_{i=0}^{k} u_{i}$ lies in the intermediate scale spaces between $L^{2}$ and $B V$, and $\sum_{i=0}^{k} v_{i}$ lies in the intermediate scale spaces between $G_{p}$ and $L^{2}$. Another application of this hierarchical decomposition is multiscale texture extraction. Indeed, $\sum_{i=0}^{k} v_{i}$ represents the textures in the scales ranging from $\lambda_{0}$ to $\lambda_{0} 2^{k}$.

3.2. Existence of Hierarchical Decomposition. The existence of our hierarchical decomposition is directly derived from the following result, actually, which can be used for original $\left(B V, G_{p}, L^{2}\right)$ decomposition by replacing $r_{i}$ with $f$, but Vese and Osher did not give proof for it in their papers.

Theorem 9. For $r_{i} \in L^{2}(i=-1,0, \ldots)$, the following minimization problem

$$
\begin{aligned}
& \inf \left\{E_{\lambda}\left(r_{i}, \lambda_{0} 2^{i+1} ; u, v\right)\right. \\
& \left.\quad=|u|_{B V}+\lambda_{0} 2^{i+1}\left\|r_{i}-u-v\right\|_{L^{2}}^{2}+\|v\|_{G_{p}}, \int_{\Omega} u=\int_{\Omega} r_{i}\right\}
\end{aligned}
$$

has a solution $(u, v)$ such that $u \in B V$ and $v \in G_{p}$.

Proof. Since $E_{\lambda}\left(r_{i}, \lambda_{0} 2^{i+1} ; u, v\right) \geq 0$ for all $u \in B V$ and $v \in G_{p}$, inf ${ }_{u \in B V, v \in G_{p}} E_{\lambda}\left(r_{i}, \lambda_{0} 2^{i+1} ; u, v\right)<+\infty$. We can find a minimizing sequence $\left\{\left(u_{n}, v_{n}\right)\right\}_{n \geq 1} \in\left(B V, G_{p}\right)$ such that $0 \leq E_{\lambda}\left(r_{i}, \lambda_{0} 2^{i+1} ; u_{n}, v_{n}\right) \leq C$ and $\int_{\Omega} u_{n}=\int_{\Omega} r_{i}$ for all $n$. Then, we have uniformly

$$
\begin{gathered}
\left|u_{n}\right|_{B V} \leq C, \\
\left\|r_{i}-u_{n}-v_{n}\right\|_{L^{2}} \leq C, \\
\left\|v_{n}\right\|_{G_{p}} \leq C .
\end{gathered}
$$

Here, the constant $C$ may be changed from line to line. By the Sobolev-Poincare inequality, we have

$$
\left\|u_{n}-\bar{u}_{n}\right\|_{L^{2}} \leq C\left|u_{n}\right|_{B V}, \quad \bar{u}_{n}=\frac{1}{|\Omega|} \int_{\Omega} u_{n}
$$

where $|\Omega|$ is the volume of $\Omega$. We thus obtain $\left\|u_{n}-\bar{u}_{n}\right\|_{L^{2}} \leq$ $C$ by (23), which implies that $u_{n}$ is uniformly bounded in $L^{2}$ since $\int_{\Omega} u_{n}=\int_{\Omega} r_{i}$ for all $n \geq 1$. Because $\Omega$ is bounded, $u_{n}$ is also uniformly bounded in $L^{1}$. By (23), we thus have

$$
\left\|u_{n}\right\|_{B V}=\left\|u_{n}\right\|_{L^{1}}+\left|u_{n}\right|_{B V} \leq C .
$$


By Theorem 3, there exists $u \in B V$ and a subsequence (still denoted by $\left.u_{n}\right)$, such that $u_{n}$ converge to $u$ in $B V$-weak* topology and weakly in $L^{2}$. In particular, by lower semicontinuity for the $B V$-weak ${ }^{*}$ topology (Theorem 4), we can obtain

$$
|u|_{B V} \leq \underset{n \rightarrow+\infty}{\lim _{n}}\left|u_{n}\right|_{B V}
$$

Since $u_{n}$ is uniformly bounded in $L^{2}$, by (23) we have that $v_{n}$ is uniformly bounded in $L^{2}$. Therefore, there exists $v \in L^{2}$ such that (up to a subsequence) $v_{n}$ converges to $v$ weakly in $L^{2}$. By weak lower semicontinuity of $L^{2}$-norm, we deduce the following property:

$$
\left\|r_{i}-u-v\right\|_{L^{2}}^{2} \leq \underset{n \rightarrow+\infty}{\lim }\left\|r_{i}-u_{n}-v_{n}\right\|_{L^{2}}^{2}
$$

For $v_{n} \in G_{p}$, by Proposition 7, there exists $\mathbf{g}_{n}=$ $\left(g_{1, n}, g_{2, n}\right) \in\left(L^{p}\right)^{2}$ such that $v_{n}=\operatorname{div}\left(\mathbf{g}_{n}\right) \in \mathscr{D}^{\prime}\left(\mathscr{D}^{\prime}\right.$ is the distribution space) and $\left\|v_{n}\right\|_{G_{p}}=\left\|\mathbf{g}_{n}\right\|_{L^{p}}$, which implies $\left\|g_{i, n}\right\|_{L^{p}} \leq C(i=1,2)$ due to $\left\|v_{n}\right\|_{G_{p}} \leq C$. Therefore, there exist $\mathbf{g}=\left(g_{1}, g_{2}\right) \in\left(L^{p}\right)^{2}$, such that, up to a subsequence, $g_{i, n}$ converges to $g_{i}$ weak ${ }^{*}$ in $L^{p}$.

We next prove that $v=\operatorname{div}(\mathbf{g}) \in G_{p}$. Let $\varphi \in \mathscr{D}(\mathscr{D}$ is the test function space); then,

$$
\int_{\Omega} v_{n} \varphi d \mathbf{x}=\int_{\Omega} \operatorname{div}\left(\mathbf{g}_{n}\right) \varphi d \mathbf{x}=-\int_{\Omega} \mathbf{g}_{n} \cdot \nabla \varphi d \mathbf{x} .
$$

Taking $n \rightarrow \infty$ (using weak $L^{2}$ topology and weak ${ }^{*} L^{p}$ topology), we obtain

$$
\int_{\Omega} \nu \varphi d \mathbf{x}=-\int_{\Omega} \mathbf{g} \cdot \nabla \varphi d \mathbf{x}=\int_{\Omega} \operatorname{div}(\mathbf{g}) \varphi d \mathbf{x} .
$$

This implies $v=\operatorname{div}(\mathbf{g}) \in \mathscr{D}^{\prime}$. And since $v \in L^{2}, v=\operatorname{div}(\mathbf{g})$ a.e. Therefore, $v \in G_{p} \cap L^{2}$. By weak* lower semicontinuity, it follows that

$$
\begin{aligned}
\|v\|_{G_{p}} & \leq\left\|\sqrt{g_{1}^{2}+g_{2}^{2}}\right\|_{L^{p}} \leq \underset{n \rightarrow+\infty}{\lim }\left\|\sqrt{g_{1, n}^{2}+g_{2, n}^{2}}\right\|_{L^{p}} \\
& =\varliminf_{n \rightarrow+\infty}\left\|v_{n}\right\|_{G_{p}} .
\end{aligned}
$$

By (26)-(30), we have

$$
E_{\lambda}\left(r_{i}, \lambda_{0} 2^{i+1} ; u, v\right) \leq \underline{\lim }_{n \rightarrow+\infty} E_{\lambda}\left(r_{i}, \lambda_{0} 2^{i+1} ; u_{n}, v_{n}\right)
$$

which implies that $(u, v)$ is a solution for $(22)$. The proof is completed.

3.3. Nontrivial Property of Hierarchical Decomposition. In this study, if the solution of (22) satisfies $u \neq 0$ or $v \neq 0$, then the decomposition $f=u+v+r$ is called the nontrivial decomposition. If $r_{i}=u_{i+1}+v_{i+1}+r_{i+1}\left(r_{-1}=f\right)$ is nontrivial for any $i \in\{-1,0,1, \ldots\}$, then the hierarchical decomposition (21) is called the nontrivial hierarchical decomposition. Conversely, if the minimization problem (22) has only zero solution, that is, $(u, v)=(0,0)$, then the decomposition $r_{i}=u_{i+1}+v_{i+1}+r_{i+1}$ of $r_{i}$ is trivial, which makes no sense for image decomposition. In what follows, we discuss the existence of the nontrivial hierarchical decomposition in (21).

Firstly, similar to (but slightly different from) Definition 5.3 of [8], we here define a new quantity $\|\cdot\|_{*}$ to measure the $L^{2}$-function, which will play a key role in our following study.

Definition 10. Let $\omega \in L^{2}$. Then, for any $h \in B V$ and $g \in$ $G_{p} \cap L^{2}$, one defines

$$
\|\omega\|_{*}=\sup _{h \in B V, g \in G_{P} \cap L^{2}} \frac{|\langle\omega, h+g\rangle|}{|h|_{B V}+\|g\|_{G_{p}}}, \quad|h|_{B V}+\|g\|_{G_{p}} \neq 0,
$$

where $\langle\cdot, \cdot\rangle$ denote $L^{2}$ inner product.

By the definition of $\|\cdot\|_{*}$, we have the following results.

Proposition 11. Let $\omega \in L^{2}$. If $\int_{\Omega} \omega d \mathbf{x} \neq 0$, then $\|\omega\|_{*}=+\infty$.

Proof. For any $h \in B V, g \in G_{p} \cap L^{2}$, and $c \in \mathbb{R}$, replacing $h$ with $c+h$ and noting that $|c+h|_{B V}=|h|_{B V}$, we have

$$
\begin{aligned}
\frac{|\langle\omega, c+h+g\rangle|}{|h+c|_{B V}+\|g\|_{G_{p}}} & =\frac{\left|c \int_{\Omega} \omega d \mathbf{x}+\langle\omega, h+g\rangle\right|}{|h|_{B V}+\|g\|_{G_{p}}} \\
& \geq \frac{|c|\left|\int_{\Omega} \omega d \mathbf{x}\right|-|\langle\omega, h+g\rangle|}{|h|_{B V}+\|g\|_{G_{p}}} .
\end{aligned}
$$

By $\int_{\Omega} \omega d \mathbf{x} \neq 0$, we can deduce that

$$
\frac{|\langle\omega, c+h+g\rangle|}{|h+c|_{B V}+\|g\|_{G_{p}}} \longrightarrow+\infty \quad \text { as }|c| \longrightarrow \infty .
$$

By the definition of $\|\cdot\|_{*}$, we have

$$
\|\omega\|_{*}=\sup \frac{|\langle\omega, h+g\rangle|}{|h|_{B V}+\|g\|_{G_{p}}}=+\infty .
$$

By Theorem 9, the minimization problem (22) must have solutions. Next, simulating hierarchical $\left(B V, L^{2}\right)$ decomposition proposed by Tadmor et al. [5], we show some properties for these solutions, which will be used to demonstrate the nontrivial property for our hierarchical decomposition.

Lemma 12. Let $r_{i} \in L^{2}$. If the minimization problem (22) has a zero solution, then $\left\|r_{i}\right\|_{*} \leq 1 / \lambda_{0} 2^{i+2}$.

Proof. Since (22) has a zero solution, then for any $h \in B V$ and $g \in G_{p}$, we have

$$
E_{\lambda}\left(r_{i}, \lambda_{0} 2^{i+1} ; h, g\right) \geq E_{\lambda}\left(r_{i}, \lambda_{0} 2^{i+1} ; 0,0\right)
$$

and that is,

$$
|h|_{B V}+\lambda_{0} 2^{i+1}\left\|r_{i}-h-g\right\|_{L^{2}}^{2}+\|g\|_{G_{p}} \geq \lambda_{0} 2^{i+1}\left\|r_{i}\right\|_{L^{2}}^{2} .
$$


This inequality can be rewritten as

$$
|h|_{B V}-\lambda_{0} 2^{i+2}\left\langle r_{i}, h+g\right\rangle+\lambda_{0} 2^{i+1}\|h+g\|_{L^{2}}^{2}+\|g\|_{G_{p}} \geq 0 .
$$

Substituting $h$ by $\varepsilon h$ and $g$ by $\varepsilon g$ in (38) and taking $\varepsilon \rightarrow 0^{+}$ and $\varepsilon \rightarrow 0^{-}$, respectively, we obtain

$$
\left|\left\langle r_{i}, h+g\right\rangle\right| \leq \frac{1}{\lambda_{0} 2^{i+2}}\left(|h|_{B V}+\|g\|_{G_{p}}\right) .
$$

By the definition of $\|\cdot\|_{*}$, we have $\left\|r_{i}\right\|_{*} \leq 1 / \lambda_{0} 2^{i+2}$.

Lemma 13. Let $r_{i} \in L^{2}$. If $\left\|r_{i}\right\|_{*}>1 / \lambda_{0} 2^{i+2}$, then the solution $\left(u_{i+1}, v_{i+1}\right)$ of $(22)$ is nonzero; that is, $u_{i+1} \neq 0$ or $v_{i+1} \neq 0$. Furthermore, $u_{i+1}, v_{i+1}$ and $r_{i+1}=r_{i}-u_{i+1}-v_{i+1}$ satisfy

$$
\begin{gathered}
\left\|r_{i+1}\right\|_{*}=\frac{1}{\lambda_{0} 2^{i+2}}, \\
\left\langle r_{i+1}, u_{i+1}+v_{i+1}\right\rangle=\frac{1}{\lambda_{0} 2^{i+2}}\left(\left|u_{i+1}\right|_{B V}+\left\|v_{i+1}\right\|_{G_{p}}\right) .
\end{gathered}
$$

Proof. The first assertion is proved directly by Lemma 12 .

Because $\left(u_{i+1}, v_{i+1}\right)$ is the solution of $(22)$, for any $h \in B V$, $g \in G_{p}$, and $\varepsilon \in \mathbb{R}$, we have

$$
\begin{aligned}
\mid u_{i+1} & +\left.\varepsilon h\right|_{B V}+\lambda_{0} 2^{i+1}\left\|r_{i+1}-\varepsilon(h+g)\right\|_{L^{2}}^{2}+\left\|v_{i+1}+\varepsilon g\right\|_{G_{p}} \\
& \geq\left|u_{i+1}\right|_{B V}+\lambda_{0} 2^{i+1}\left\|r_{i+1}\right\|_{L^{2}}^{2}+\left\|v_{i+1}\right\|_{G_{p}}
\end{aligned}
$$

By the triangle inequality, we obtain

$$
\begin{aligned}
\mid u_{i+1}+ & \left.\varepsilon h\right|_{B V}+\lambda_{0} 2^{i+1}\left\|r_{i+1}-\varepsilon(h+g)\right\|_{L^{2}}^{2}+\left\|v_{i+1}+\varepsilon g\right\|_{G_{p}} \\
\leq & \left(\left|u_{i+1}\right|_{B V}+|\varepsilon||h|_{B V}\right)+\lambda_{0} 2^{i+1}\left\|r_{i+1}-\varepsilon(h+g)\right\|_{L^{2}}^{2} \\
& +\left(\left\|v_{i+1}\right\|_{G_{p}}+|\varepsilon|\|g\|_{G_{p}}\right) .
\end{aligned}
$$

So, the inequality (41) is changed into

$$
\begin{gathered}
|\varepsilon||h|_{B V}+\lambda_{0} 2^{i+1}\left\|r_{i+1}-\varepsilon(h+g)\right\|_{L^{2}}^{2}+|\varepsilon|\|g\|_{G_{p}} \\
\geq \lambda_{0} 2^{i+1}\left\|r_{i+1}\right\|_{L^{2}}^{2} .
\end{gathered}
$$

Expanding the second term on left side of the last inequality, we can obtain

$$
\begin{gathered}
|\varepsilon||h|_{B V}+\varepsilon^{2} \lambda_{0} 2^{i+1}\|(h+g)\|_{L^{2}}^{2}+|\varepsilon|\|g\|_{G_{p}} \\
\geq \varepsilon \lambda_{0} 2^{i+2}\left\langle r_{i+1}, h+g\right\rangle .
\end{gathered}
$$

Dividing both sides of the last inequality by $\varepsilon>0$ and taking $\varepsilon \rightarrow 0^{+}$, we obtain

$$
|h|_{B V}+\|g\|_{G_{p}} \geq \lambda_{0} 2^{i+2}\left\langle r_{i+1}, h+g\right\rangle .
$$

Dividing both sides of (44) by $\varepsilon<0$ and taking $\varepsilon \rightarrow 0^{-}$, we also obtain

$$
|h|_{B V}+\|g\|_{G_{p}} \geq-\lambda_{0} 2^{i+2}\left\langle r_{i+1}, h+g\right\rangle .
$$

The inequalities (45) and (46) imply that

$$
|h|_{B V}+\|g\|_{G_{p}} \geq \lambda_{0} 2^{i+2}\left|\left\langle r_{i+1}, h+g\right\rangle\right| .
$$

By definition of $\|\cdot\|_{*}$, we have

$$
\left\|r_{i+1}\right\|_{*} \leq \frac{1}{\lambda_{0} 2^{i+2}} .
$$

Let $\varepsilon \in(-1,1)$. Replacing $(h, g)$ with $\left(u_{i+1}, v_{i+1}\right)$ in the inequality (41), we have

$$
\begin{aligned}
\mid u_{i+1} & +\left.\varepsilon u_{i+1}\right|_{B V}+\lambda_{0} 2^{i+1}\left\|r_{i+1}-\varepsilon\left(u_{i+1}+v_{i+1}\right)\right\|_{L^{2}}^{2} \\
& +\left\|v_{i+1}+\varepsilon v_{i+1}\right\|_{G_{p}} \\
= & (1+\varepsilon)\left|u_{i+1}\right|_{B V}+\lambda_{0} 2^{i+1}\left\|r_{i+1}-\varepsilon\left(u_{i+1}+v_{i+1}\right)\right\|_{L^{2}}^{2} \\
& +(1+\varepsilon)\left\|v_{i+1}\right\|_{G_{p}} \\
\geq & \left|u_{i+1}\right|_{B V}+\lambda_{0} 2^{i+1}\left\|r_{i+1}\right\|_{L^{2}}^{2}+\left\|v_{i+1}\right\|_{G_{p}} .
\end{aligned}
$$

So,

$$
\begin{gathered}
\varepsilon\left|u_{i+1}\right|_{B V}+\varepsilon^{2} \lambda_{0} 2^{i+1}\left\|u_{i+1}+v_{i+1}\right\|_{L^{2}}^{2}+\varepsilon\left\|v_{i+1}\right\|_{G_{p}} \\
\geq \varepsilon \lambda_{0} 2^{i+2}\left\langle r_{i+1}, u_{i+1}+v_{i+1}\right\rangle .
\end{gathered}
$$

Dividing both sides of the last inequality by $|\varepsilon|$ and then taking $\varepsilon \rightarrow 0^{+}$and $\varepsilon \rightarrow 0^{-}$, respectively, we obtain the equality (40):

$$
\lambda_{0} 2^{i+2}\left\langle r_{i+1}, u_{i+1}+v_{i+1}\right\rangle=\left|u_{i+1}\right|_{B V}+\left\|v_{i+1}\right\|_{G_{p}} .
$$

So,

$$
\lambda_{0} 2^{i+2}\left|\left\langle r_{i+1}, u_{i+1}+v_{i+1}\right\rangle\right|=\left|u_{i+1}\right|_{B V}+\left\|v_{i+1}\right\|_{G_{p}},
$$

which, due to $u_{i+1} \neq 0$ or $v_{i+1} \neq 0$, implies

$$
\frac{\left|\left\langle r_{i+1}, u_{i+1}+v_{i+1}\right\rangle\right|}{\left|u_{i+1}\right|_{B V}+\left\|v_{i+1}\right\|_{G_{p}}}=\frac{1}{\lambda_{0} 2^{i+2}} \text {. }
$$

By definition of $\|\cdot\|_{*}$ and (48), we have $\left\|r_{i+1}\right\|_{*}=1 / \lambda_{0} 2^{i+2}$.

Theorem 14. Let $f \in L^{2}$ with $\int_{\Omega} f d \mathbf{x} \neq 0$, and $\left(u_{i+1}, v_{i+1}\right)$ is the solution of (22). Then, for any initial scale $\lambda_{0}>0$, the decomposition $r_{i}=u_{i+1}+v_{i+1}+r_{i+1}$ is nontrivial for any $i \epsilon$ $\{-1,0,1, \ldots\}$. In other words, any hierarchical decomposition of $f$ given in (21) is nontrivial.

Proof. Since $\int_{\Omega} f d \mathbf{x} \neq 0$, we have $\|f\|_{*}=+\infty$ by Proposition 11. By Lemma 13, the decomposition $f=r_{-1}=u_{0}+v_{0}+r_{0}$ is 
nontrivial, and $\left\|r_{0}\right\|_{*}=1 /\left(2 \lambda_{0}\right)$. Because $\left\|r_{0}\right\|_{*}=1 /\left(2 \lambda_{0}\right)>$ $1 /\left(2^{2} \lambda_{0}\right)$, again by Lemma 13 , the decomposition $r_{0}=u_{1}+$ $v_{1}+r_{1}$ is nontrivial, and $\left\|r_{1}\right\|_{*}=1 /\left(2^{2} \lambda_{0}\right)>1 /\left(2^{3} \lambda_{0}\right)$ which means such nontrivial decomposition can continue.

For the $i$ th decomposition, by Lemma 13, we have $\left\|r_{i-1}\right\|_{*}=1 /\left(\lambda_{0} 2^{i}\right)>1 /\left(\lambda_{0} 2^{i+1}\right)$ which means the $i$ th decomposition $r_{i}=u_{i+1}+v_{i+1}+r_{i+1}$ is nontrivial. In conclusion, any hierarchical decomposition of $f$ given in (21) is nontrivial when $\int_{\Omega} f d \mathbf{x} \neq 0$.

Remark 15. By Theorems 9 and 14, we can deduce that for any $L^{2}$-function $f$ with $\int_{\Omega} f d \mathbf{x} \neq 0$, there must be a nontrivial hierarchical decomposition. This result is much significant for image hierarchical decomposition. In general, a digital image $f$ is a nonnegative $L^{2}$-function with $\int_{\Omega} f d \mathbf{x} \neq 0$, so any hierarchical $\left(B V, G_{p}, L^{2}\right)$ decomposition of $f$ must be nontrivial.

3.4. Convergence of Hierarchical Decomposition. For the hierarchical decomposition given in (21), we have the following convergence result (Theorem 17) in the $L^{2}$ topology, which is similar to the convergence result of hierarchical $\left(B V, L^{2}\right)$ decomposition proposed by Tadmor, Nezzar, and Vese (see Theorem 2.2 in [5] for details). To prove Theorem 17, we need the following lemma.

Lemma 16. If $r_{i} \in L^{2}$, then there are $\widehat{u} \in B V$, and $\widehat{v} \in$ $L^{2} \subset G_{p}$ so that $E_{\lambda}\left(r_{i}, \lambda_{0} 2^{i+1} ; \widehat{u}, \widehat{v}\right) \leq C$, where $C$ is a constant independent of $\lambda_{0} 2^{i+1}$.

Proof. By [19], there exists a unique solution for ROF model (1), denoted by

$$
(\widehat{u}, \widehat{v})=\arg \inf \left\{J\left(r_{i} ; u, v\right)=|u|_{B V}+\|v\|_{L^{2}}, r_{i}=u+v\right\}
$$

such that $\widehat{u} \in B V, \widehat{v} \in L^{2} \subset G_{p}$. Therefore, we can deduce that

$$
\begin{aligned}
E_{\lambda}\left(r_{i}, \lambda_{0} 2^{i+1} ; \widehat{u}, \widehat{v}\right) & =|\widehat{u}|_{B V}+2^{i+1} \lambda_{0}\left\|r_{i}-\widehat{u}-\widehat{v}\right\|_{L^{2}}^{2}+\|\widehat{v}\|_{G_{p}} \\
& =|\widehat{u}|_{B V}+\|\hat{v}\|_{G_{p}} \\
& \leq|\widehat{u}|_{B V}+C_{1}\|\hat{v}\|_{L^{2}}=C,
\end{aligned}
$$

where $C$ clearly does not depend on $\lambda_{0} 2^{i+1}$.

Theorem 17. Let $f \in L^{2}$. Then, the hierarchical decomposition given in (21) satisfies

$$
\left\|f-\sum_{i=-1}^{k}\left(u_{i+1}+v_{i+1}\right)\right\|_{L^{2}}=\left\|r_{k+1}\right\|_{L^{2}} \longrightarrow 0, \quad \text { as } k \longrightarrow \infty .
$$

In addition, the following "energy" estimate holds:

$$
\begin{aligned}
& \sum_{i=-1}^{\infty} \frac{1}{\lambda_{0} 2^{i+1}}\left(\left|u_{i+1}\right|_{B V}+\left\|v_{i+1}\right\|_{G_{p}}\right) \\
& +\sum_{i=-1}^{\infty}\left(\left\|u_{i+1}+v_{i+1}\right\|_{L^{2}}^{2}\right)=\|f\|_{L^{2}}^{2} .
\end{aligned}
$$

Proof. By Lemma 16, there exist $\widehat{u} \in B V$ and $\widehat{v} \in G_{p}$, such that $E_{\lambda}\left(r_{i}, \lambda_{0} 2^{i+1} ; \widehat{u}, \widehat{v}\right) \leq C$, where $C$ does not depend on $\lambda_{0} 2^{i+1}$. Since $\left(u_{i+1}, v_{i+1}\right)$ is a solution of (22), we have

$$
E_{\lambda}\left(r_{i}, \lambda_{0} 2^{i+1} ; u_{i+1}, v_{i+1}\right) \leq E_{\lambda}\left(r_{i}, \lambda_{0} 2^{i+1} ; \widehat{u}, \widehat{v}\right) \leq C .
$$

Thus,

$$
\lambda_{0} 2^{i+1}\left\|r_{i}-u_{i+1}-v_{i+1}\right\|_{L^{2}}^{2} \leq E_{\lambda}\left(r_{i}, \lambda_{0} 2^{i+1} ; u_{i+1}, v_{i+1}\right) \leq C,
$$

which, by $r_{i+1}=r_{i}-u_{i+1}-v_{i+1}$, implies

$$
\left\|r_{i+1}\right\|_{L^{2}}^{2} \leq \frac{C}{\lambda_{0} 2^{i+1}}, \quad i=-1,0,1, \ldots
$$

By $r_{k+1}=f-\sum_{i=-1}^{k}\left(u_{i+1}+v_{i+1}\right)$, we have

$$
\begin{array}{r}
\left\|f-\sum_{i=-1}^{k}\left(u_{i+1}+v_{i+1}\right)\right\|_{L^{2}}^{2}=\left\|r_{k+1}\right\|_{L^{2}}^{2} \leq \frac{C}{\lambda_{0} 2^{k+1}}, \\
k=-1,0,1, \ldots .
\end{array}
$$

Therefore, $\left\|f-\sum_{i=-1}^{k}\left(u_{i+1}+v_{i+1}\right)\right\|_{L^{2}} \rightarrow 0$ as $k \rightarrow \infty$. The proof of the first assertion is completed.

Next, we prove the second assertion that is, (57). Since $r_{i}=r_{i+1}+\left(u_{i+1}+v_{i+1}\right)$, we obtain

$$
\left\|r_{i}\right\|_{L^{2}}^{2}=\left\|r_{i+1}\right\|_{L^{2}}^{2}+\left\|u_{i+1}+v_{i+1}\right\|_{L^{2}}^{2}+2\left\langle r_{i+1}, u_{i+1}+v_{i+1}\right\rangle .
$$

By (40), (62) can be rewritten as

$$
\begin{aligned}
\left\|r_{i}\right\|_{L^{2}}^{2} & -\left\|r_{i+1}\right\|_{L^{2}}^{2}-\left\|u_{i+1}+v_{i+1}\right\|_{L^{2}}^{2} \\
& =2\left\langle r_{i+1}, u_{i+1}+v_{i+1}\right\rangle \\
& =\frac{1}{\lambda_{0} 2^{i+1}}\left(\left|u_{i+1}\right|_{B V}+\left\|v_{i+1}\right\|_{G_{p}}\right) .
\end{aligned}
$$

Since

$$
\begin{aligned}
& \sum_{i=-1}^{k}\left(\left\|r_{i}\right\|_{L^{2}}^{2}-\left\|r_{i+1}\right\|_{L^{2}}^{2}-\left\|u_{i+1}+v_{i+1}\right\|_{L^{2}}^{2}\right) \\
& =\sum_{i=-1}^{k}\left(\left\|r_{i}\right\|_{L^{2}}^{2}-\left\|r_{i+1}\right\|_{L^{2}}^{2}\right)-\sum_{i=-1}^{k}\left(\left\|u_{i+1}+v_{i+1}\right\|_{L^{2}}^{2}\right) \\
& =\left\|r_{-1}\right\|_{L^{2}}^{2}-\left\|r_{k+1}\right\|_{L^{2}}^{2}-\sum_{i=-1}^{k}\left(\left\|u_{i+1}+v_{i+1}\right\|_{L^{2}}^{2}\right) \\
& =\|f\|_{L^{2}}^{2}-\left\|r_{k+1}\right\|_{L^{2}}^{2}-\sum_{i=-1}^{k}\left(\left\|u_{i+1}+v_{i+1}\right\|_{L^{2}}^{2}\right),
\end{aligned}
$$




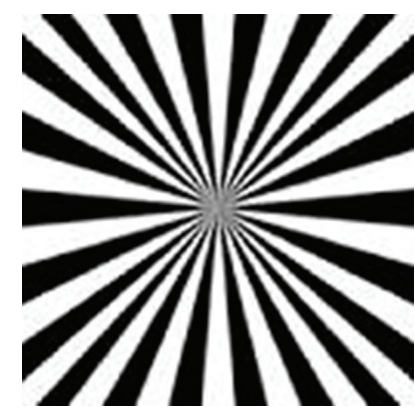

(a)

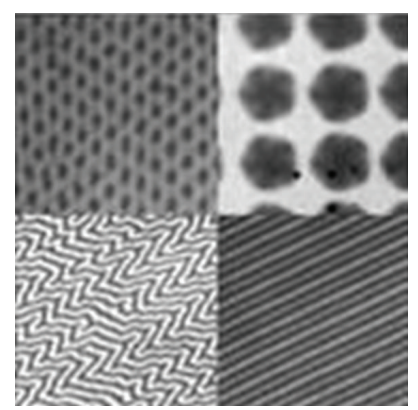

(b)

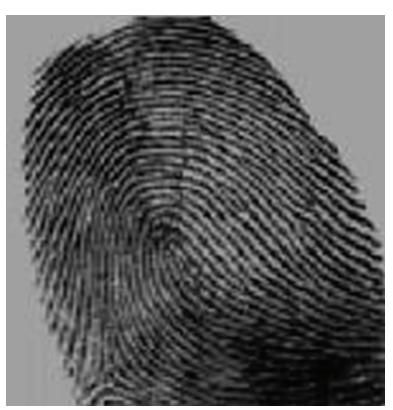

(c)

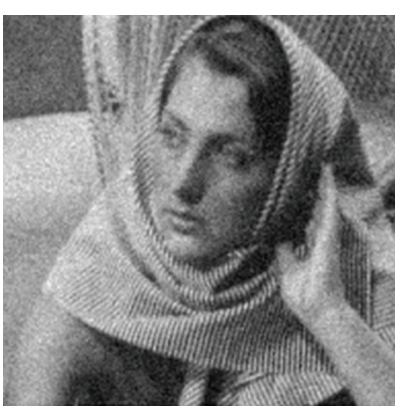

(d)

FIGURE 1: Test images. Left to right: (a) and (b) two synthetic textured images; (c) fingerprint image; (d) a portion of noisy Barbana image which generated by adding Gaussian noise with standard deviation 20 to the clean data.

summing up both sides of (63), we obtain

$$
\begin{gathered}
\sum_{i=-1}^{k} \frac{1}{\lambda_{0} 2^{i+1}}\left(\left|u_{i+1}\right|_{B V}+\left\|v_{i+1}\right\|_{G_{p}}\right) \\
+\sum_{i=-1}^{k}\left(\left\|u_{i+1}+v_{i+1}\right\|_{L^{2}}^{2}\right) \\
=\|f\|_{L^{2}}^{2}-\left\|r_{k+1}\right\|_{L^{2}}^{2} .
\end{gathered}
$$

By $\lim _{k \rightarrow \infty}\left\|r_{k+1}\right\|_{L^{2}} \rightarrow 0$, we have

$$
\begin{aligned}
& \sum_{i=-1}^{\infty} \frac{1}{\lambda_{0} 2^{i+1}}\left(\left|u_{i+1}\right|_{B V}+\left\|v_{i+1}\right\|_{G_{p}}\right) \\
& +\sum_{i=-1}^{\infty}\left(\left\|u_{i+1}+v_{i+1}\right\|_{L^{2}}^{2}\right)=\|f\|_{L^{2}}^{2} .
\end{aligned}
$$

Equation (57) can be seen as the $L^{2}$-energy decomposition of $f$ in our hierarchical decomposition. In addition, the multiscale nature of our hierarchical extraction can be quantified in terms of this energy decomposition.

\section{Numerical Implementation}

In this section, we present the details of numerical implementation for our hierarchical $\left(B V, G_{p}, L^{2}\right)$ decomposition:

$$
\begin{aligned}
& \left(u_{k+1}, v_{k+1}\right) \\
& =\operatorname{arginf}\left\{|u|_{B V}+\lambda_{0} 2^{k+1}\left\|r_{k}-u-v\right\|_{L^{2}}^{2}+\|v\|_{G_{p}},\right. \\
& \left.\int_{\Omega} u=\int_{\Omega} r_{k}\right\}, \quad k=-1,0,1, \ldots
\end{aligned}
$$

Taking $v=\operatorname{div}(\mathbf{g})=\operatorname{div}\left(g_{1}, g_{2}\right)$, we obtain the following equivalent formulation of (67) in terms of $u, g_{1}$, and $g_{2}$ :

$$
\begin{aligned}
\left(u_{k+1},\left[g_{1}\right]_{k+1},\left[g_{2}\right]_{k+1}\right) & \\
=\operatorname{arginf} & \left\{|u|_{B V}+\lambda_{0} 2^{k+1}\left\|r_{k}-u-\operatorname{div}(\mathbf{g})\right\|_{L^{2}}^{2}\right. \\
& \left.+\|\mathbf{g}\|_{L^{p}}, \int_{\Omega} u=\int_{\Omega} r_{k}\right\},
\end{aligned}
$$

where $\mathbf{g}_{k+1}=\left(\left[g_{1}\right]_{k+1},\left[g_{2}\right]_{k+1}\right)$.
Minimizing the energy in (68) with respect to $u, g_{1}$ and $g_{2}$ yields the following Euler-Lagrange equations:

$$
\begin{gathered}
-\operatorname{div}\left(\frac{\nabla u}{|\nabla u|}\right)-\lambda_{0} 2^{k+2}\left(r_{k}-u-\frac{\partial}{\partial x} g_{1}-\frac{\partial}{\partial y} g_{2}\right)=0 \\
\lambda_{0} 2^{k+2} \frac{\partial}{\partial x}\left(r_{k}-u-\frac{\partial}{\partial x} g_{1}-\frac{\partial}{\partial y} g_{2}\right) \\
+\left(\left\|\sqrt{g_{1}^{2}+g_{2}^{2}}\right\|_{L^{p}}\right)^{1-p}\left(\sqrt{g_{1}^{2}+g_{2}^{2}}\right)^{p-2} g_{1}=0 \\
\lambda_{0} 2^{k+2} \frac{\partial}{\partial y}\left(r_{k}-u-\frac{\partial}{\partial x} g_{1}-\frac{\partial}{\partial y} g_{2}\right) \\
+\left(\left\|\sqrt{g_{1}^{2}+g_{2}^{2}}\right\|_{L^{p}}\right)^{1-p}\left(\sqrt{g_{1}^{2}+g_{2}^{2}}\right)^{p-2} g_{2}=0
\end{gathered}
$$

If the exterior normal to the boundary $\partial \Omega$ is denoted by $\left(n_{x}, n_{y}\right)$, then the associated boundary conditions for $u, g_{1}$, and $g_{2}$ are

$$
\begin{gathered}
\nabla u \cdot\left(n_{x}, n_{y}\right)=0 \\
\left(r_{k}-u-\frac{\partial}{\partial x} g_{1}-\frac{\partial}{\partial y} g_{2}\right) n_{x}=0 \\
\left(r_{k}-u-\frac{\partial}{\partial x} g_{1}-\frac{\partial}{\partial y} g_{2}\right) n_{y}=0 .
\end{gathered}
$$

Equation (69) with boundary condition (72) implies that $\int_{\Omega} u=\int_{\Omega} r_{k}$ holds. Indeed, by taking the integral for each side of (69) and using the Gaussian formula, we obtain

$$
\int_{\Omega}\left(r_{k}-u-\operatorname{div}(\mathbf{g})\right)=0
$$

Since $v=\operatorname{div}(\mathbf{g}) \in G_{p}$, by Proposition 8 , we have $\int_{\Omega} \operatorname{div}(\mathbf{g})=$ 0 . Therefore, $\int_{\Omega} u=\int_{\Omega} r_{k}$.

We solve (69)-(71) by the alternating algorithm. For each equation, we adopt gradient decent method. To simplify the presentation, we introduce the notation

$$
H\left(g_{1}, g_{2}\right)=\left(\left\|\sqrt{g_{1}^{2}+g_{2}^{2}}\right\|_{L^{p}}\right)^{1-p}\left(\sqrt{g_{1}^{2}+g_{2}^{2}}\right)^{p-2} .
$$




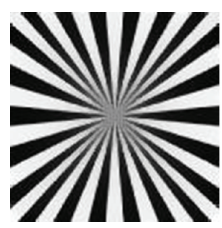

$u_{0}$

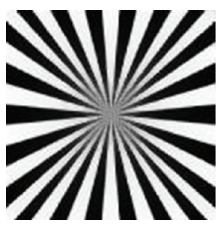

$\sum_{i=0}^{1} u_{i}$

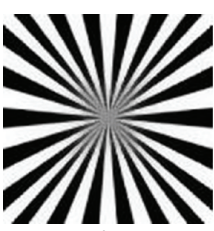

$\sum_{i=0}^{2} u_{i}$

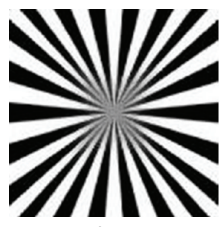

$\sum_{i=0}^{3} u_{i}$

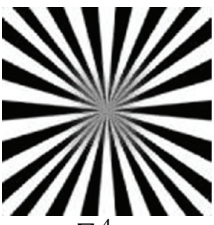

$\sum_{i=0}^{4} u_{i}$

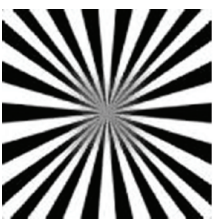

$\sum_{i=0}^{5} u_{i}$

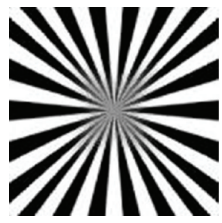

$\sum_{i=0}^{6} u_{i}$

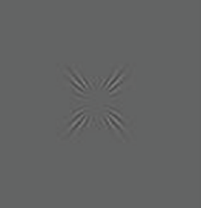

$v_{0}+100$

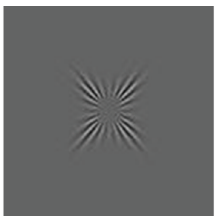

$\sum_{i=0}^{1} v_{i}+100$

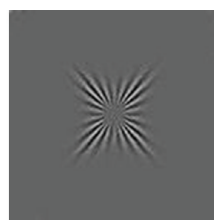

$\sum_{i=0}^{2} v_{i}+100$

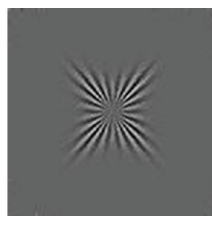

$\sum_{i=0}^{3} v_{i}+100$

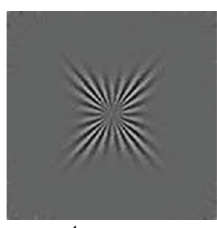

$\sum_{i=0}^{4} v_{i}+100$

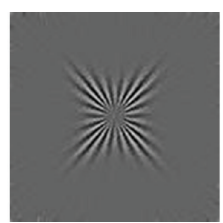

$\sum_{i=0}^{5} v_{i}+100$

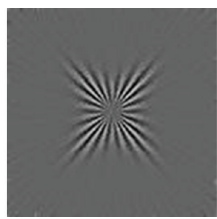

$\sum_{i=0}^{6} v_{i}+100$

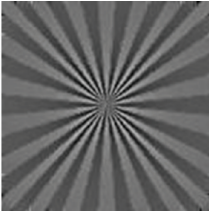

$r_{0}+100$

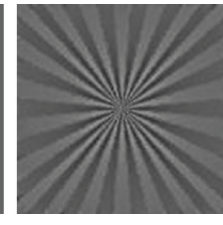

$r_{1}+100$

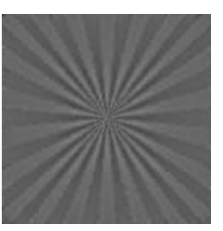

$r_{2}+100$

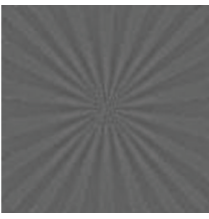

$r_{3}+100$

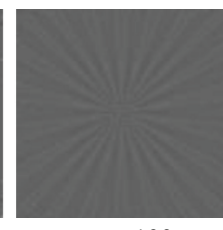

$r_{4}+100$

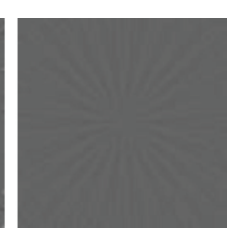

$r_{5}+100$

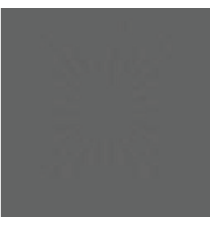

$r_{6}+100$
FIGURE 2: Hierarchical decomposition of a synthetic image for 7 steps.

The details are as follows:

(i) fixed $\left(g_{1}, g_{2}\right)$, find the solution $u$ of

$$
\frac{\partial u}{\partial t}=\operatorname{div}\left(\frac{\nabla u}{|\nabla u|}\right)+\lambda_{0} 2^{k+2}\left(r_{k}-u-\frac{\partial}{\partial x} g_{1}-\frac{\partial}{\partial y} g_{2}\right)
$$

(ii) fixed $u$, find the solution $g_{1}, g_{2}$ of

$$
\begin{aligned}
\frac{\partial g_{1}}{\partial t}= & -\lambda_{0} 2^{k+2} \frac{\partial}{\partial x}\left(r_{k}-u-\frac{\partial}{\partial x} g_{1}-\frac{\partial}{\partial y} g_{2}\right) \\
& -H\left(g_{1}, g_{2}\right) g_{1}, \\
\frac{\partial g_{2}}{\partial t}= & -\lambda_{0} 2^{k+2} \frac{\partial}{\partial y}\left(r_{k}-u-\frac{\partial}{\partial x} g_{1}-\frac{\partial}{\partial y} g_{2}\right) \\
& -H\left(g_{1}, g_{2}\right) g_{2},
\end{aligned}
$$

with the initial conditions $g_{1}^{0}=-\left(\left(1 /\left(\lambda_{0} 2^{k+2}\right)\right)\left(r_{k, x} /\right.\right.$ $\left.\left.\left|\nabla r_{k}\right|\right)\right), g_{2}^{0}=-\left(\left(1 /\left(\lambda_{0} 2^{k+2}\right)\right)\left(r_{k, y} /\left|\nabla r_{k}\right|\right)\right)$, respectively.

We use a simple explicit finite difference scheme to solve (77)-(78). The image domain $\Omega$ is discretized by the space steps $\Delta x$ and $\Delta y$. Then, the grid is defined as

$$
\begin{gathered}
(x, y)=\left(x_{i}, y_{j}\right)=(i \Delta x, j \Delta y), \\
i=0,1,2, \ldots, m, \quad j=0,1,2, \ldots, n .
\end{gathered}
$$

We denote the time step by $\Delta t$, and $t_{n}=n \Delta t(n=0,1,2, \ldots)$. Let $u_{i, j}^{n}$ be the value of $u(x, y, t)$ at the grid $\left(x_{i}, y_{j}, t_{n}\right)$. In order to compute the right hand side of (77)-(78), we denote

$$
\begin{aligned}
\Delta_{+}^{x} u_{i, j}^{n}=\frac{u_{i+1, j}^{n}-u_{i, j}^{n}}{\Delta x}, & \Delta_{-}^{x} u_{i, j}^{n}=\frac{u_{i, j}^{n}-u_{i-1, j}^{n}}{\Delta x}, \\
\Delta_{+}^{y} u_{i, j}^{n}=\frac{u_{i, j+1}^{n}-u_{i, j}^{n}}{\Delta y}, & \Delta_{-}^{y} u_{i, j}^{n}=\frac{u_{i, j}^{n}-u_{i, j-1}^{n}}{\Delta y}, \\
\Delta_{0}^{x} u_{i, j}^{n}=\frac{\left(\Delta_{+}^{x}+\Delta_{-}^{x}\right) u_{i, j}^{n}}{2}, & \Delta_{0}^{y} u_{i, j}^{n}=\frac{\left(\Delta_{+}^{y}+\Delta_{-}^{y}\right) u_{i, j}^{n}}{2} .
\end{aligned}
$$

Then, (77)-(78) can be approximated by the following discretizations (to remove the singularity when $|\nabla u|=0$ and $\sqrt{g_{1}^{2}+g_{2}^{2}}=0$, we introduce a regularity parameter $\left.\varepsilon^{2}\right)$ :

$$
\begin{aligned}
u_{i, j}^{n+1}=u_{i, j}^{n}+\Delta t & K_{i, j}^{n}+\lambda_{0} 2^{k+2} \\
& \left.\times\left(\left[r_{k}\right]_{i, j}-u_{i, j}^{n}-\Delta_{0}^{x}\left[g_{1}\right]_{i, j}^{n}-\Delta_{0}^{y}\left[g_{2}\right]_{i, j}^{n}\right)\right],
\end{aligned}
$$

with the initial condition

$$
u_{i, j}^{0}=\left[r_{k}\right]_{i, j},
$$




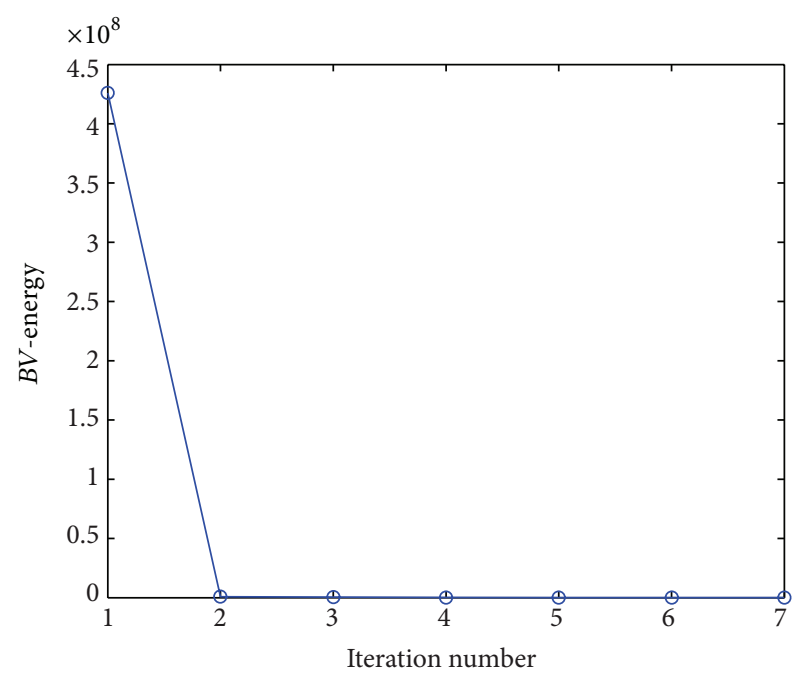

(a)

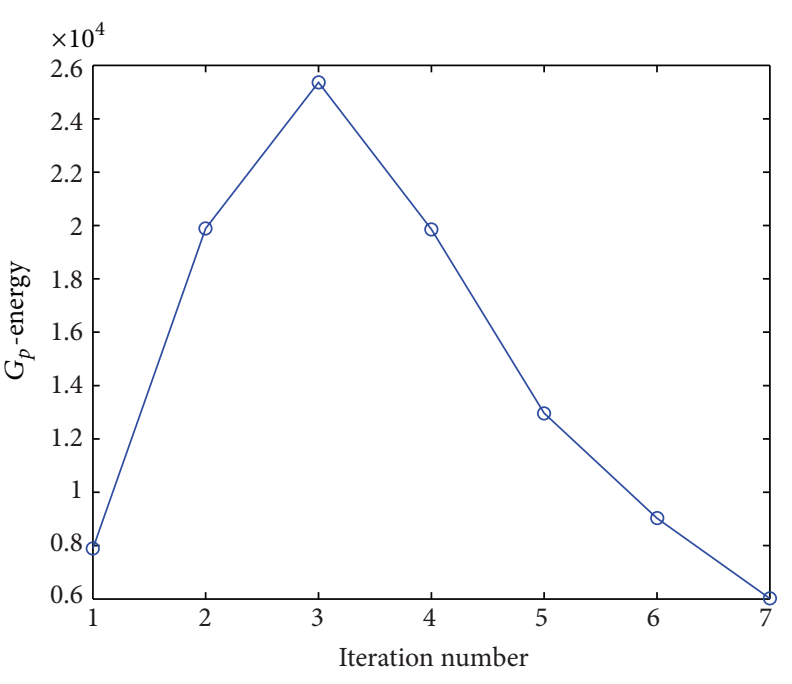

(b)

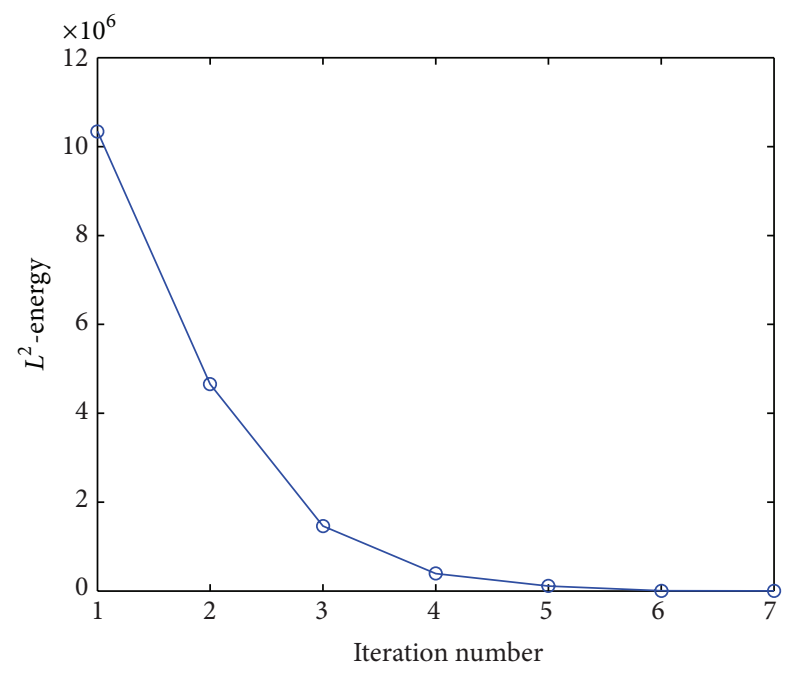

(c)

FIGURE 3: Energy plots of three components. (a) The $B V$-energy of $u_{i}$. (b) The $G_{p}$-energy of $v_{i}$. (c) The $L^{2}$-energy of $r_{i}$.

where $K_{i, j}^{n}$ is the curvature of the level set of $u$ at the grid $\left(x_{i}, y_{j}, n \Delta t\right)$, defined by

$$
\begin{aligned}
& K_{i, j}^{n}= \Delta_{+}^{x}\left(\frac{\Delta_{-}^{x} u_{i, j}^{n}}{\sqrt{\left(\Delta_{-}^{x} u_{i, j}^{n}\right)^{2}+\left(\Delta_{-}^{x} u_{i, j}^{n}\right)^{2}+\varepsilon^{2}}}\right) \\
&+\Delta_{+}^{y}\left(\frac{\Delta_{-}^{y} u_{i, j}^{n}}{\sqrt{\left(\Delta_{-}^{x} u_{i, j}^{n}\right)^{2}+\left(\Delta_{-}^{x} u_{i, j}^{n}\right)^{2}+\varepsilon^{2}}}\right), \\
& {\left[g_{1}\right]_{i, j}^{n+1}=} {\left[g_{1}\right]_{i, j}^{n} } \\
&-\Delta t\left[\lambda _ { 0 } 2 ^ { k + 2 } \Delta _ { 0 } ^ { x } \left(\left[r_{k}\right]_{i, j}-u_{i, j}^{n}-\Delta_{0}^{x}\left[g_{1}\right]_{i, j}^{n}\right.\right. \\
&\left.\left.-\Delta_{0}^{y}\left[g_{2}\right]_{i, j}^{n}\right)+H_{i, j}^{n}\left[g_{1}\right]_{i, j}^{n}\right],
\end{aligned}
$$

$$
\begin{aligned}
{\left[g_{2}\right]_{i, j}^{n+1}=} & {\left[g_{2}\right]_{i, j}^{n} } \\
& -\Delta t\left[\lambda _ { 0 } 2 ^ { k + 2 } \Delta _ { 0 } ^ { y } \left(\left[r_{k}\right]_{i, j}-u_{i, j}^{n}-\Delta_{0}^{x}\left[g_{1}\right]_{i, j}^{n}\right.\right. \\
& \left.\left.\quad-\Delta_{0}^{y}\left[g_{2}\right]_{i, j}^{n}\right)+H_{i, j}^{n}\left[g_{2}\right]_{i, j}^{n}\right],
\end{aligned}
$$

with the initial condition

$$
\begin{aligned}
& {\left[g_{1}\right]_{i, j}^{0}=-\frac{1}{\lambda_{0} 2^{k+2}} \frac{\Delta_{0}^{x}\left[r_{k}\right]_{i, j}}{\sqrt{\left(\Delta_{0}^{x}\left[r_{k}\right]_{i, j}\right)^{2}+\left(\Delta_{0}^{x}\left[r_{k}\right]_{i, j}\right)^{2}+\varepsilon^{2}}},} \\
& {\left[g_{2}\right]_{i, j}^{0}=-\frac{1}{\lambda_{0} 2^{k+2}} \frac{\Delta_{0}^{y}\left[r_{k}\right]_{i, j}}{\sqrt{\left(\Delta_{0}^{x}\left[r_{k}\right]_{i, j}\right)^{2}+\left(\Delta_{0}^{x}\left[r_{k}\right]_{i, j}\right)^{2}+\varepsilon^{2}}},}
\end{aligned}
$$

where $H_{i, j}^{n}=H\left(\left[g_{1}\right]_{i, j}^{n},\left[g_{2}\right]_{i, j}^{n}\right)$. 


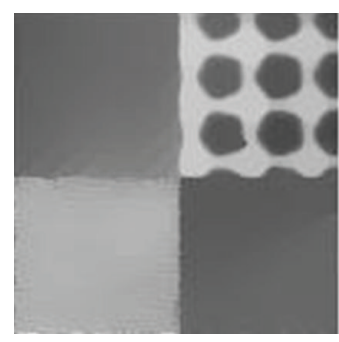

$u_{0}$

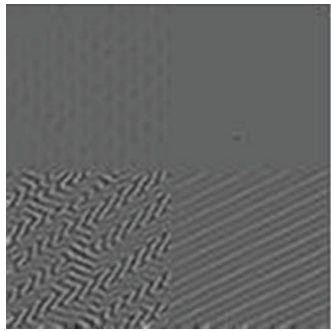

$\sum_{i=0}^{2} v_{i}+100$

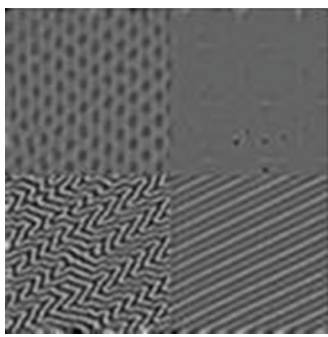

$\sum_{i=0}^{6} v_{i}+100$

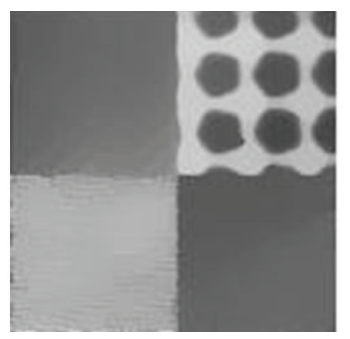

$\sum_{i=0}^{8} u_{i}$

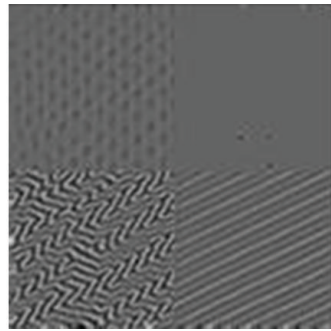

$\sum_{i=0}^{3} v_{i}+100$

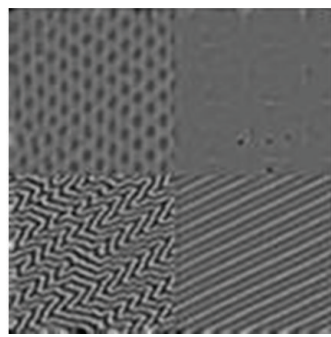

$\sum_{i=0}^{7} v_{i}+100$

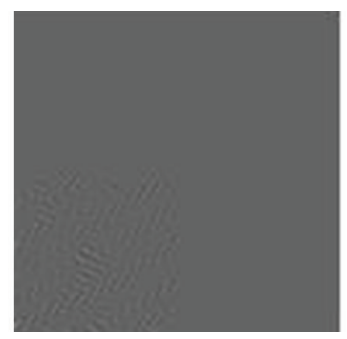

$v_{0}+100$

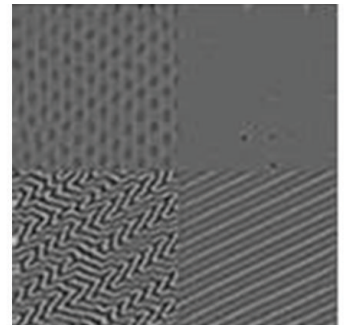

$\sum_{i=0}^{4} v_{i}+100$

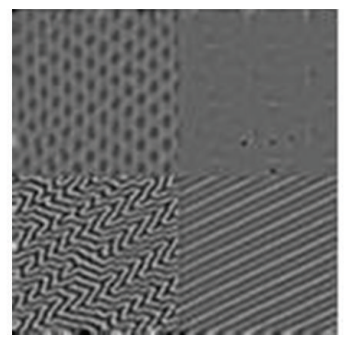

$\sum_{i=0}^{8} v_{i}+100$

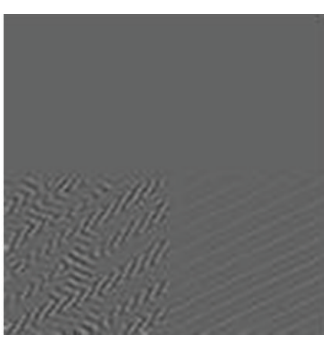

$\sum_{i=0}^{1} v_{i}+100$

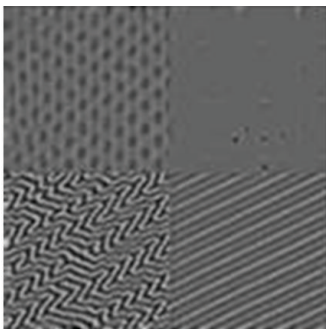

$\sum_{i=0}^{5} v_{i}+100$

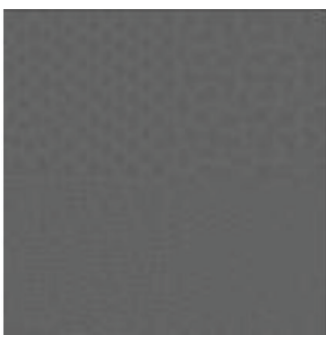

$r_{8}+100$

FIGURE 4: Multiscale texture extraction using hierarchical decomposition of a synthetic image for 9 steps.

\section{Numerical Results}

We present four numerical examples in this section to demonstrate the efficiency of multiscale texture extraction and image representation using the proposed hierarchical $\left(B V, G_{p}, L^{2}\right)$ decomposition for textured images. Test images, shown in Figure 1, are two synthetic images and two real images. In all experiments, we take the time step $\Delta t=0.05$, the space step $\Delta x=\Delta y=1$, the initial scale $\lambda_{0}=0.005$, and the regular parameter $\varepsilon^{2}=10^{-9}$.

For the choice of $p$, by the theoretical analysis in Section 2 , we have that $G_{p}$-norms are weaker than $G$-norm for any $1 \leq p<\infty$. So, any choice of $p$ with $1 \leq p<\infty$ is suitable. Here, similar to what was done by Vese and Osher in $[6,14]$, we tested the model $(68)$ with different values of $p$; our observation is that results are very similar, while the case of $p=1$ yields faster calculations per iteration. Thus, we set $p=1$ in the following. We note in passing that some different approaches based on duality principle have been proposed, such as $[21,22]$, to solve (67) with $p=\infty$. We here adopt the method introduced by Vese and Osher because this study is following their work in $[6,14]$.

(i) Image hierarchical $\left(B V, G_{p}, L^{2}\right)$ decomposition: Figure 2 shows the hierarchical decomposition results for a synthetic textured image for 7 steps. The first column shows the cartoon components of the initial image in different scales. We can see that these cartoon components are very little different visually. This phenomenon is compatible with the theory of causality of scale space. The second column shows the "textures+100" (plus a constant for illustration purposes) of the image in different scales. It is clear that the textures can be gently extracted by increasing the value of scale parameter $\lambda_{0} 2^{i+1}$, because this image involves the textures of different scales: coarser textures correspond to the smaller scales, while smoother textures correspond to the larger scales. The third column shows "residuals+100," from which we can clearly see that some textures and edges are swept into these residual components when the value of scale parameter $\lambda_{0} 2^{i+1}$ is smaller, and then, they are gradually swept out and absorbed by $u_{i}$ and $v_{i}$ by increasing the value of the scale parameter $\lambda_{0} 2^{i+1}$. Figure 3 shows the plots of the $B V$-energy of $u_{i}, G_{p^{-}}$ energy of $v_{i}$, and $L^{2}$-energy of $r_{i}$, respectively.

(ii) Multiscale texture extraction: Figure 4 shows the results of multiscale texture extraction using hierarchical decomposition for another synthetic textured image for 9 steps. The first two images show the initial and final cartoon components which have little visual difference; this phenomenon is identical with the results of the first experiment. The next nine images show the texture components in different 


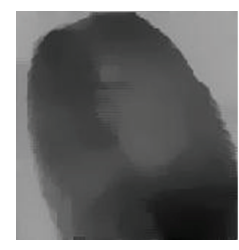

$u_{0}$

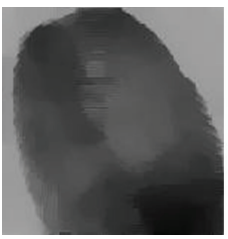

$\sum_{i=0}^{1} u_{i}$

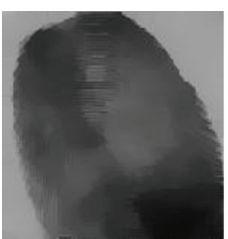

$\sum_{i=0}^{2} u_{i}$

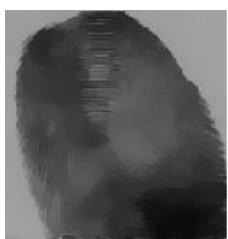

$\sum_{i=0}^{3} u_{i}$

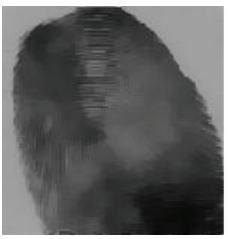

$\sum_{i=0}^{4} u_{i}$

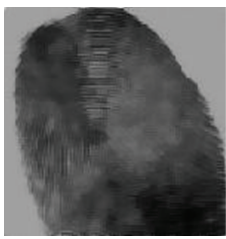

$\sum_{i=0}^{5} u_{i}$

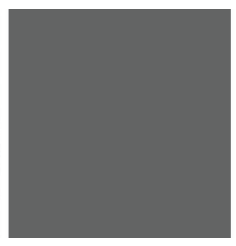

$v_{0}+100$

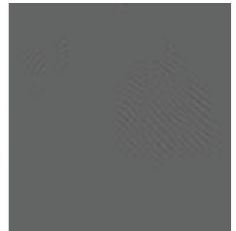

$\sum_{i=0}^{1} v_{i}+100$

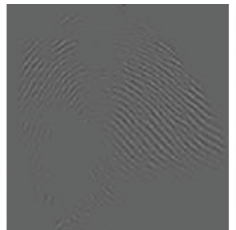

$\sum_{i=0}^{2} v_{i}+100$

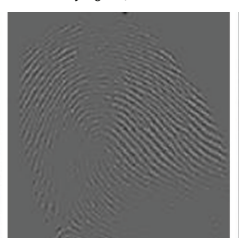

$\sum_{i=0}^{3} v_{i}+100$

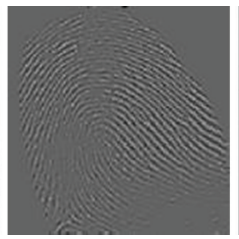

$\sum_{i=0}^{4} v_{i}+100$

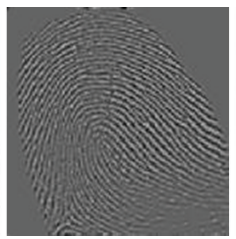

$\sum_{i=0}^{5} v_{i}+100$

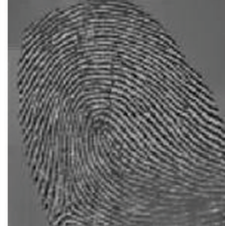

$r_{0}+100$

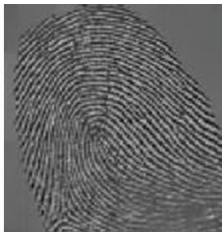

$r_{1}+100$

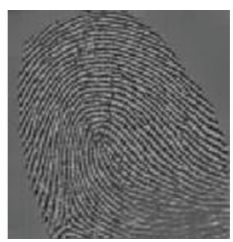

$r_{2}+100$

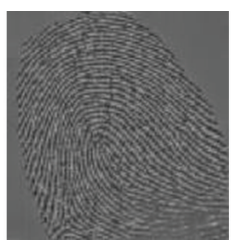

$r_{3}+100$

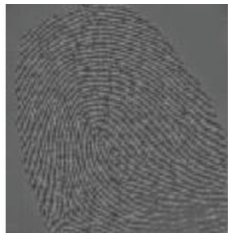

$r_{4}+100$

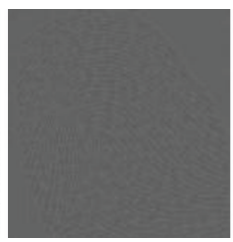

$r_{5}+100$

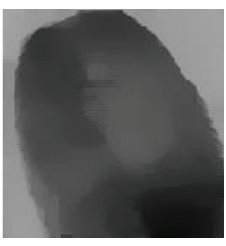

$u_{0}+v_{0}$

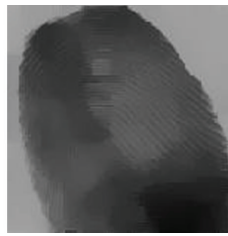

$\sum_{i=0}^{1}\left(u_{i}+v_{i}\right)$

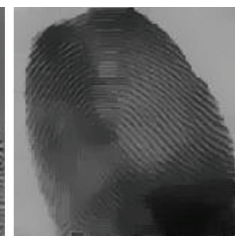

$\sum_{i=0}^{2}\left(u_{i}+v_{i}\right)$

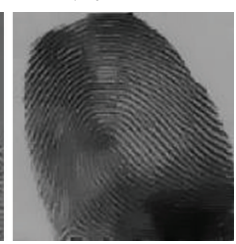

$\sum_{i=0}^{3}\left(u_{i}+v_{i}\right)$

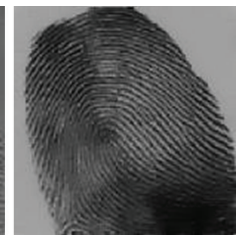

$\sum_{i=0}^{4}\left(u_{i}+v_{i}\right)$

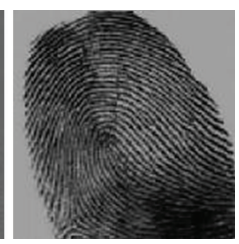

$\sum_{i=0}^{5}\left(u_{i}+v_{i}\right)$

FIGURE 5: Multiscale texture extraction and image representation using hierarchical decomposition of a fingerprint for 6 steps.

scales, which can be used as the results of multiscale texture extraction for this synthetic textured image. We remark that the larger scale textures are gradually resolved from the residual in terms of the increasing scale.

(iii) Multiscale texture extraction and image representation: Figure 5 shows the results of multiscale texture extraction and image representation using hierarchical decomposition of a fingerprint for 6 steps. The second column of this figure shows the extracted texture in different scales. The $\sum_{i=0}^{k}\left(u_{i}+v_{i}\right)$ s are shown in the last column of this figure, which can be used as a multiscale representation of the original image. We can clearly see that, from top to bottom of this column, an additional amount of blurred texture is resolved in terms of the refined scaling for edges.

(iv) Multiscale image representation for noisy textured image: Figure 6 shows the hierarchical decomposition results of a noisy Barbana for 6 steps. The last column of this figure shows $\sum_{i=0}^{k}\left(u_{i}+v_{i}\right)$ s which can be seen as restored images in different scales. Clearly, when the value of $k$ is smaller, such as $k=0,1$, there are a few textures and noises in the restored images, much of which is swept into residual components. When $k=2,3$, some textures of the image are recovered on the headscarf of Barbana while removing the smaller scale noises from the entire image. If we continue the 


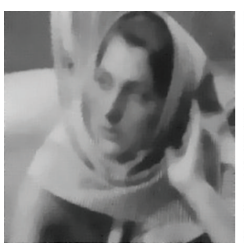

$u_{0}$

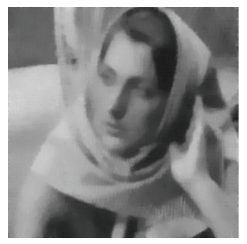

$\sum_{i=0}^{1} u_{i}$

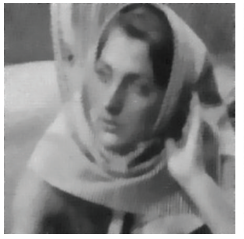

$\sum_{i=0}^{2} u_{i}$

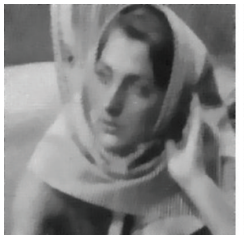

$\sum_{i=0}^{3} u_{i}$

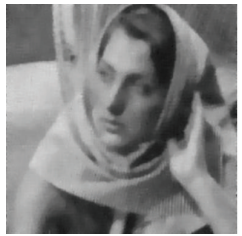

$\sum_{i=0}^{4} u_{i}$

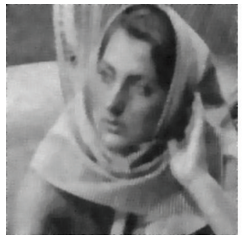

$\sum_{i=0}^{5} u_{i}$

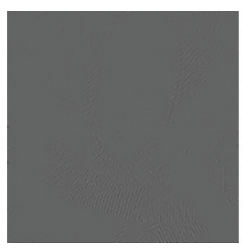

$v_{0}+100$

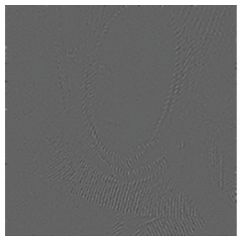

$\sum_{i=0}^{1} v_{i}+100$

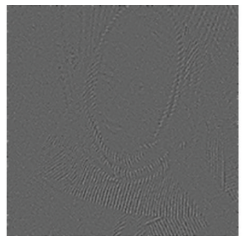

$\sum_{i=0}^{2} v_{i}+100$

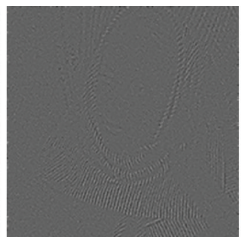

$\sum_{i=0}^{3} v_{i}+100$

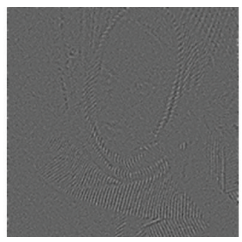

$\sum_{i=0}^{4} v_{i}+100$

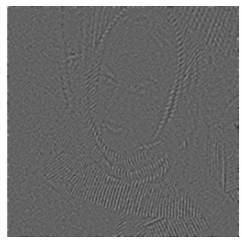

$\sum_{i=0}^{5} v_{i}+100$

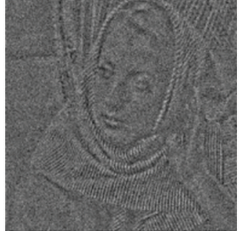

$r_{0}+100$

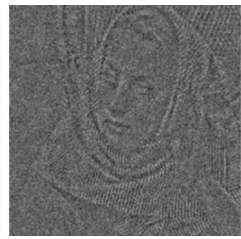

$r_{1}+100$

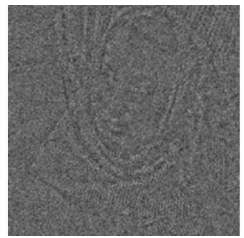

$r_{2}+100$

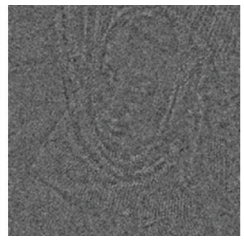

$r_{3}+100$

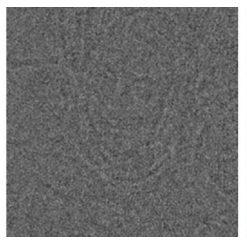

$r_{4}+100$

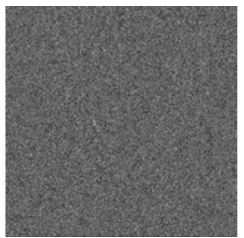

$r_{5}+100$

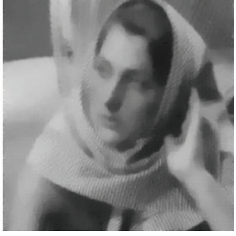

$u_{0}+v_{0}$

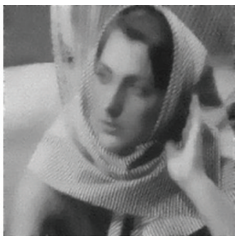

$\sum_{i=0}^{1}\left(u_{i}+v_{i}\right)$

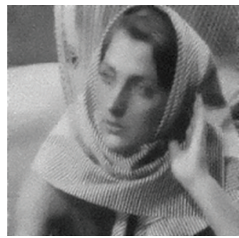

$\sum_{i=0}^{2}\left(u_{i}+v_{i}\right)$

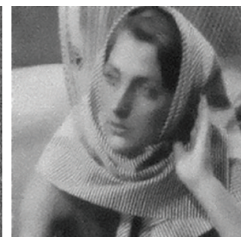

$\sum_{i=0}^{3}\left(u_{i}+v_{i}\right)$

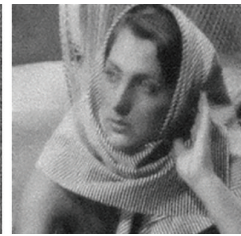

$\sum_{i=0}^{4}\left(u_{i}+v_{i}\right)$

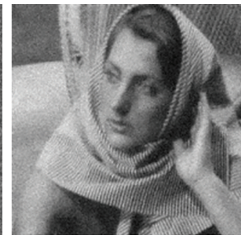

$\sum_{i=0}^{5}\left(u_{i}+v_{i}\right)$

FIGURE 6: Multiscale image representation using hierarchical decomposition of a noisy Barbana for 6 steps.

decomposition into smaller scales, then noise will reappear in the $\sum_{i=0}^{k}\left(u_{i}+v_{i}\right)$ components, since the refined scales reach the same scales of the noise itself. From the last column of this figure, we can obtain restored image from noisy Barbana in different scales according to our requirements.

\section{Conclusions}

In this paper, in order to achieve multiscale image representation and texture extraction for textured image, we presented a hierarchical $\left(B V, G_{p}, L^{2}\right)$ decomposition model which combines the idea of hierarchical decomposition introduced by
Tadmor et al. with the $\left(B V, G_{p}, L^{2}\right)$ decomposition proposed by Vese et al. In addition, we proved the existence and the convergence of the hierarchical decomposition, and the nontrivial property of this decomposition is also discussed. But the uniqueness of this hierarchical decomposition has not been proved in this paper. The authors will be concerned about this problem in the successive research.

\section{References}

[1] G. Aubert and P. Kornprobst, Mathematical Problems in Image Processing, Springer, New York, NY, USA, 2001. 
[2] T. Chan, J. Shen, and L. Vese, "Variational PDE models in image processing," Notices of the American Mathematics Society, vol. 50, no. 1, pp. 14-26, 2003.

[3] L. I. Rudin, S. Osher, and E. Fatemi, "Nonlinear total variation based noise removal algorithms," Physica D, vol. 60, no. 1-4, pp. 259-268, 1992.

[4] S. Osher, A. Sole, and L. Vese, "Image decomposition and restoration using total variation minimization and the $\mathrm{H}^{1}$," Multiscale Modeling and Simulation, vol. 1, no. 3, pp. 349-370, 2003.

[5] E. Tadmor, S. Nezzar, and L. Vese, "A multiscale image representation using hierarchical (BV; $\mathrm{L}^{2}$ ) decompositions," Multiscale Modeling and Simulation, vol. 2, no. 4, pp. 554-579, 2003.

[6] L. Vese and S. Osher, "Modeling textures with total variation minimization and oscillating patterns in image processing," Journal of Scientific Computing, vol. 19, no. 1-3, pp. 553-572, 2003.

[7] J. Shen, "Piecewise $\mathrm{H}^{-1}+\mathrm{H}^{0}+\mathrm{H}^{1}$ images and the MumfordShah-Sobolev model for segmented image decomposition," Applied Mathematics Research Express, vol. 4, pp. 143-167, 2005.

[8] T. M. Le and L. Vese, "Image decomposition using total variation and $\operatorname{div}(\mathrm{BMO}), "$ Multiscale Modeling and Simulation, vol. 4, no. 2, pp. 390-423, 2005.

[9] C. W. Lu and G. X. Song, "Image decomposition using adaptive regularization and div (BMO)," Journal of Systems Engineering and Electronics, vol. 22, no. 2, pp. 358-364, 2011.

[10] A. Chambolle, R. DeVore, N. Lee, and B. Lucier, "Nonlinear wavelet image processing: variational problems, compression, and noise removal through wavelet shrinkage," IEEE Transactions on Image Processing, vol. 7, no. 3, pp. 319-335, 1998.

[11] Z. Jin and X. Yang, "Analysis of a new variational model for multiplicative noise removal," Journal of Mathematical Analysis and Applications, vol. 362, no. 2, pp. 415-426, 2010.

[12] I. Daubechies and G. Teschke, "Variational image restoration by means of wavelets: simultaneous decomposition, deblurring, and denoising," Applied and Computational Harmonic Analysis, vol. 19, no. 1, pp. 1-16, 2005.

[13] E. Tadmor, S. Nezzar, and L. Vese, "Multiscale hierarchical decomposition of images with applications to deblurring, denoising and segmentation," Communications in Mathematical Sciences, vol. 6, no. 2, pp. 281-307, 2008.

[14] L. Vese and S. Osher, "Image denoising and decomposition with total variation minimization and oscillatory functions," Journal of Mathematical Imaging and Vision, vol. 20, no. 1-2, pp. 7-18, 2004.

[15] Y. Meyer, Oscillating Patterns in Image Processing and Nonlinear Evolution Equations, American Mathematical Society, 2001.

[16] K. H. Liang and T. Tjahjadi, "Adaptive scale fixing for multiscale texture segmentation," IEEE Transactions on Image Processing, vol. 15, no. 1, pp. 249-256, 2006.

[17] Y. F. Pu and J. L. Zhou, "A novel approach for multi-scale texture segmentation based on fractional differential," International Journal of Computer Mathematics, vol. 88, no. 1, pp. 58-78, 2011.

[18] L. Evans and R. Gariepy, Measure Theory and Fine Properties of Functions, CRC Press, Boca Raton, Fla, USA, 1992.

[19] A. Chambolle and P. L. Lions, "Image recovery via total variation minimization and related problems," Numerische Mathematik, vol. 76, no. 2, pp. 167-188, 1997.

[20] G. Aubert and J. Aujol, "Modeling very oscillating signals. Application to image processing," Applied Mathematics and Optimization, vol. 51, no. 2, pp. 163-182, 2005.
[21] J. F. Aujol, G. Aubert, L. Blanc-Féraud, and A. Chambolle, "Image decomposition into a bounded variation component and an oscillating component," Journal of Mathematical Imaging and Vision, vol. 22, no. 1, pp. 71-88, 2005.

[22] A. Chambolle and T. Pock, "A first-order primal-dual algorithm for convex problems with applications to imaging," Journal of Mathematical Imaging and Vision, vol. 40, no. 1, pp. 120-145, 2011. 


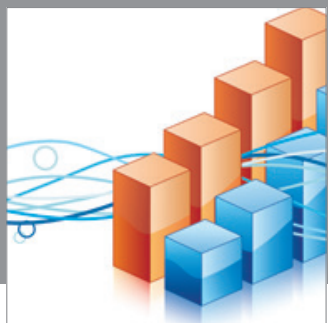

Advances in

Operations Research

mansans

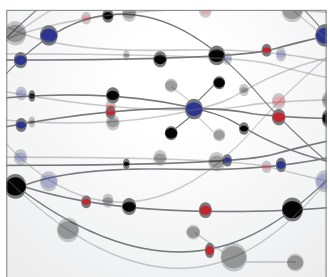

The Scientific World Journal
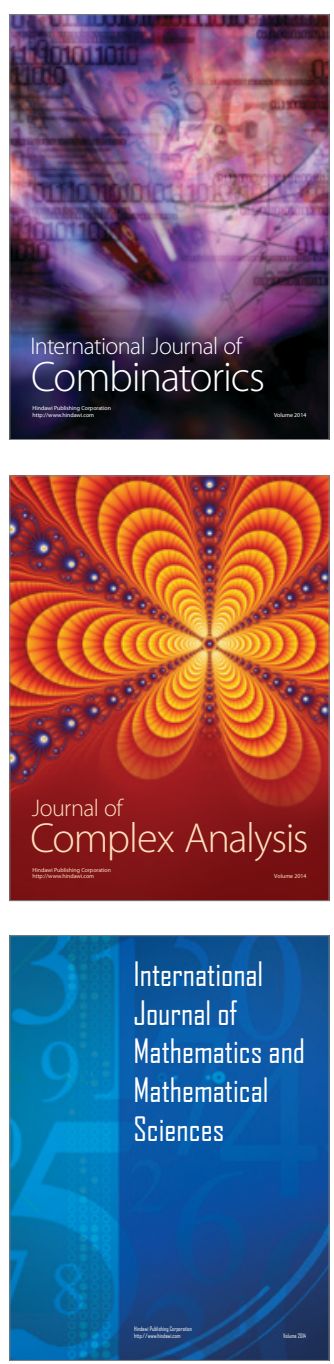
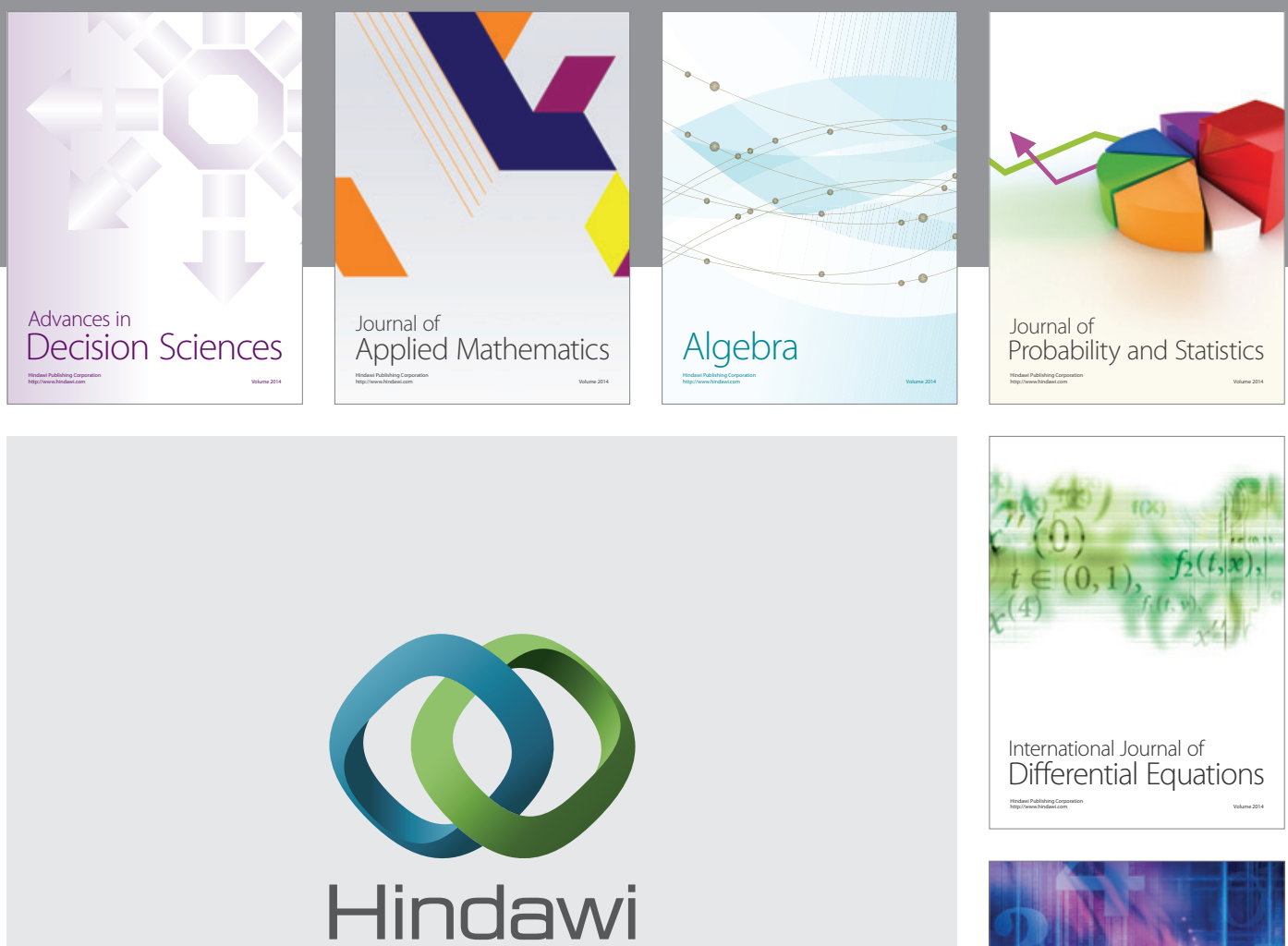

Submit your manuscripts at http://www.hindawi.com
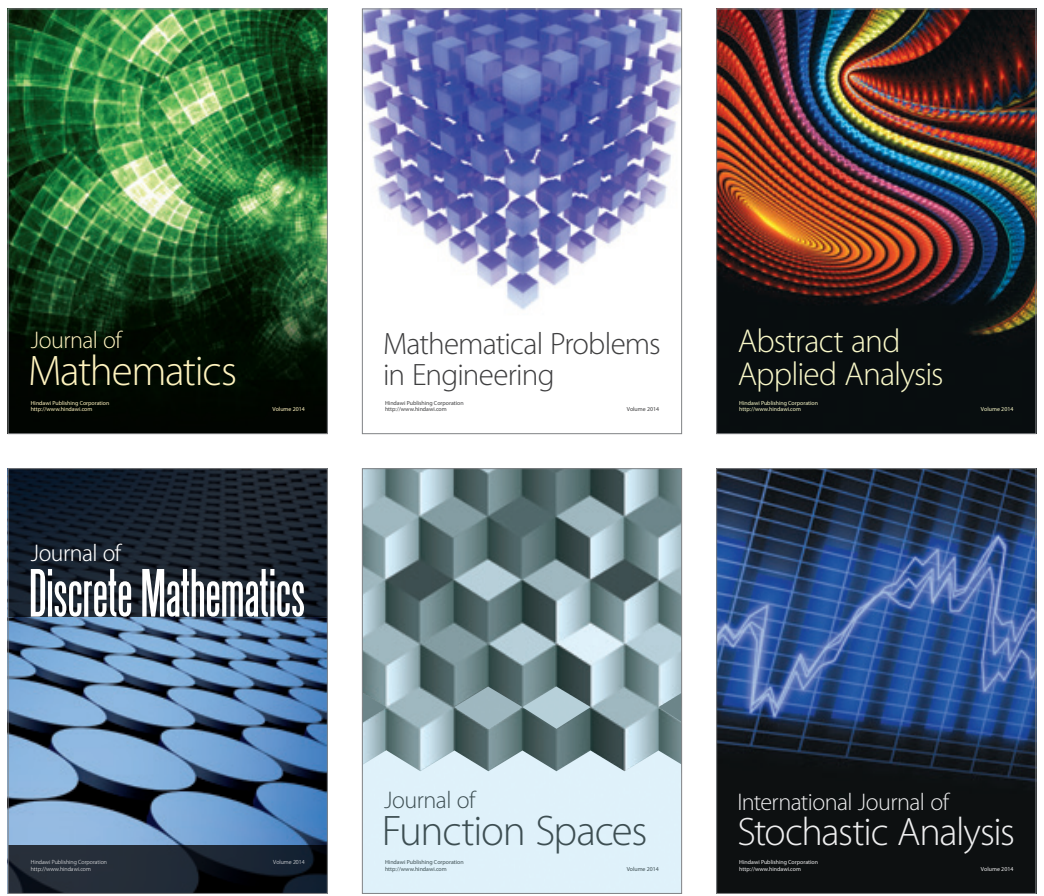

Journal of

Function Spaces

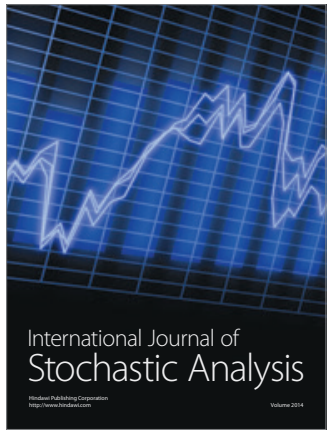

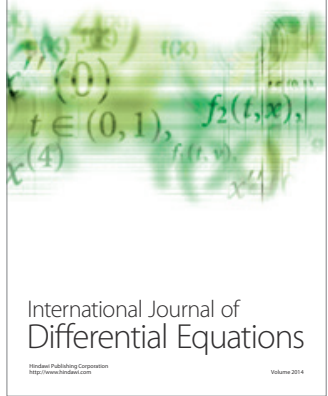
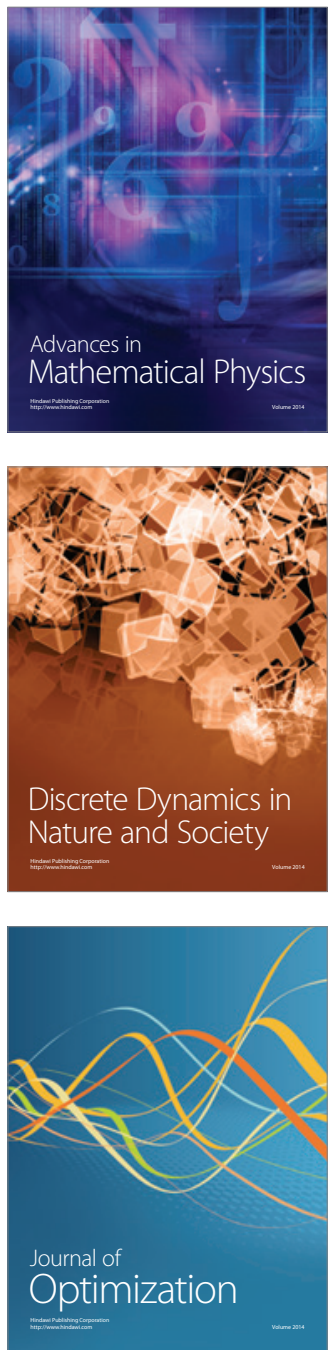